TRANSACTIONS OF THE

AMERICAN MATHEMATICAL SOCIETY

Volume 360, Number 2, February 2008, Pages 643-669

S 0002-9947(07)04139-6

Article electronically published on June 25, 2007

\title{
EXISTENCE OF RENORMALIZED SOLUTIONS TO NONLINEAR ELLIPTIC EQUATIONS WITH TWO LOWER ORDER TERMS AND MEASURE DATA
}

\author{
OLIVIER GUIBÉ AND ANNA MERCALDO
}

\begin{abstract}
In this paper we prove the existence of a renormalized solution to a class of nonlinear elliptic problems whose prototype is

$$
\begin{cases}-\triangle_{p} u-\operatorname{div}\left(c(x)|u|^{\gamma}\right)+b(x)|\nabla u|^{\lambda}=\mu & \text { in } \Omega, \\ u=0 & \text { on } \partial \Omega\end{cases}
$$

where $\Omega$ is a bounded open subset of $\mathbb{R}^{N}, N \geq 2, \triangle_{p}$ is the so-called $p$-Laplace operator, $1<p<N, \mu$ is a Radon measure with bounded variation on $\Omega$, $0 \leq \gamma \leq p-1,0 \leq \lambda \leq p-1$, and $|c|$ and $b$ belong to the Lorentz spaces $L^{\frac{N}{p-1}, r}(\Omega), \frac{N}{p-1} \leq r \leq+\infty$, and $L^{N, 1}(\Omega)$, respectively. In particular we prove the existence under the assumptions that $\gamma=\lambda=p-1,|c|$ belongs to the Lorentz space $L^{\frac{N}{p-1}, r}(\Omega), \frac{N}{p-1} \leq r<+\infty$, and $\|c\|_{L^{\frac{N}{p-1}, r}(\Omega)}$ is small enough.
\end{abstract}

\section{INTRODUCTION}

In this paper we consider nonlinear elliptic problems whose prototype is

$$
\begin{cases}-\triangle_{p} u-\operatorname{div}\left(c(x)|u|^{\gamma}\right)+b(x)|\nabla u|^{\lambda}=\mu & \text { in } \Omega, \\ u=0 & \text { on } \partial \Omega,\end{cases}
$$

where $\Omega$ is a bounded open subset of $\mathbb{R}^{N}, N \geq 2, \triangle_{p}$ is the so-called $p$-Laplace operator, $p$ is a real number such that $1<p<N, \mu$ is a Radon measure with bounded variation on $\Omega, 0 \leq \gamma \leq p-1,0 \leq \lambda \leq p-1$, and $|c|$ and $b$ belong to the Lorentz spaces $L^{\frac{N}{p-1}, r}(\Omega), \frac{N}{p-1} \leq r \leq+\infty$, and $L^{N, 1}(\Omega)$, respectively.

We are interested in existence results for renormalized solutions to (1.1).

We have proved such an existence result in [GM], when $\mu$ is a Radon measure with bounded variation on $\Omega, \gamma=\lambda=p-1$, and $\|c\|_{L^{\frac{N}{p-1}, r}(\Omega)}, r<+\infty$, is large and $\|b\|_{L^{N, 1}(\Omega)}$ is small enough; the existence of a renormalized solution is also obtained, without assumption on the smallness of the norms of the coefficients, when $\gamma$ or $\lambda$ are less than $p-1$.

In the present paper we investigate the counterpart of the existence result given in GM], that is, we prove the existence of a renormalized solution when $\mu$ is a Radon measure with bounded variation on $\Omega, \gamma=p-1, \lambda=p-1,\|b\|_{L^{N, 1}(\Omega)}$ is

Received by the editors December 16, 2003 and, in revised form, May 23, 2005 and August 2, 2005.

2000 Mathematics Subject Classification. Primary 35J60; Secondary 35A35, 35J25, 35R10.

Key words and phrases. Existence, nonlinear elliptic equations, noncoercive problems, measures data. 
large and $\|c\|_{L^{\frac{N}{p-1}, r}(\Omega)}, r<+\infty$, is small. The case $\gamma<p-1$ (and $\lambda \leq p-1$ ) is also studied.

The main features of (1.1) are both the fact that the operator has two lower order terms, which produce a lack of coercivity, and the right-hand side which is a measure.

Let us assume that the operator has no lower order terms, i.e. $b=c=0$; in this case the difficulties in studying problem (1.1) are due only to the right-hand side $\mu$.

Simple examples (the Laplace operator in a ball, i.e. $p=2, b=0, c=0$, and $\mu$ the Dirac mass in the center) show that, in general, the solution of (1.1) does not belong to the space $W_{l o c}^{1,1}(\Omega)$. Thus it is necessary to change the classical framework of Sobolev spaces in order to prove existence results.

In the linear case, i.e. $p=2$, Stampacchia introduced a notion of solution of problem (1.1) defined by "duality" ([St] for which he proved the existence and the uniqueness. He also proved that such a solution satisfies the equation in the distributional sense and it belongs to $W_{0}^{1, q}(\Omega)$ for every $q<N /(N-1)$. Unfortunately, Stampacchia's arguments cannot be extended to the nonlinear case except in the case where $p=2$ as shown in $\mathrm{M} 2$.

The first attempt in studying the nonlinear case was done by Boccardo and Gallouët ([BG1, [BG2]), who proved, under the assumption $p>2-\frac{1}{N}$, the existence of a solution which satisfies the equation in the distributional sense and which belongs to $W_{0}^{1, q}(\Omega)$ for every $q<\frac{N(p-1)}{N-1}$. Let us explicitly remark that the assumption on $p$ implies that $\frac{N(p-1)}{N-1}>1$.

The next step consists of finding an "extra condition" on the distributional solutions of (1.1) in order to prove both existence and uniqueness results. This is done by introducing two equivalent notions of solution: the notion of entropy solution in BBGGPV], BGO and the notion of the renormalized solution in [LM], M1. These settings were, however, limited to the case of measure in $L^{1}(\Omega)$ or in $L^{1}(\Omega)+W^{-1, p^{\prime}}(\Omega)$. The case of a general measure with bounded total variation was studied in DMOP], where the notion of renormalized solution has been extended to this case and an existence result is proved.

The effect of the two terms $b(x)|\nabla u|^{\lambda}$ and $-\operatorname{div}\left(c(x)|u|^{\gamma}\right)$ is a lack of coercivity of the operator.

In the linear case, i.e. $p=2, \gamma=\lambda=1$, Stampacchia proved the existence and the uniqueness of a "duality" solution, if 0 is not in the spectrum of the operator. Such condition is verified if, for example, $\|c\|_{L^{\frac{N}{p-1}}(\Omega)}$ or $\|b\|_{L^{N}(\Omega)}$ is small enough. The case of a nonlinear operator was studied in [D], where a term $b(x)|\nabla u|^{\lambda}$ is considered, and in [DPo1, where both terms $-\operatorname{div}\left(c(x)|u|^{\gamma}\right)$ and $b(x)|\nabla u|^{\lambda}$ are considered; in these papers the existence of a solution which satisfies the equation in the distributional sense is proved.

The effects of both the right-hand side a measure and the lower order term $b(x)|\nabla u|^{\lambda}$ were studied in BMMP3, where the existence of a renormalized solution is proved.

Existence results for entropy solutions are proved by Boccardo in $\mathrm{B}$ when the operator has a lower order term of the type $-\operatorname{div}(c(x) u)$. Moreover, in the nonlinear case when the operator has a lower order term of the type $-\operatorname{div}\left(c(x)|u|^{\gamma}\right)$ and the 
right-hand side $\mu$ belongs to $L^{1}(\Omega)$, the existence of a renormalized solution is proved in BGu1, BGu2].

Finally let us explain the restriction $p<N$. If $p$ is greater than the dimension $N$ of the ambient space, then, by the Sobolev embedding theorem and duality arguments, the space of measures with bounded variation on $\Omega$ is a subset of $W^{-1, p^{\prime}}(\Omega)$, so that the existence of solutions in $W_{0}^{1, p}(\Omega)$ was proved by Stampacchia in the linear case, i.e. $p=2, \gamma=\lambda=1$ (see also $\mathrm{Dr}$ ) and by [DPo2] (see also G2 for a different proof).

Uniqueness results for renormalized solutions can be found in BMMP2, when the datum $\mu$ belongs to $L^{1}(\Omega)+W^{-1, p^{\prime}}(\Omega)$ and the operator has a lower order term of the type $b(x)|\nabla u|^{\lambda}$ (see BMMP4 for the case where $\mu$ belongs to $W^{-1, p^{\prime}}(\Omega)$ ) and in [BGu1], BGu2] when $\mu$ belongs to $L^{1}(\Omega)$ and a lower order term of the type $-\operatorname{div}\left(c(x)|u|^{\gamma}\right)$ is considered (see also G1 for further uniqueness results).

In the present paper we consider operators where both the two lower order terms - $\operatorname{div}\left(c(x)|u|^{\gamma}\right)$ and $b(x)|\nabla u|^{\lambda}$ appear without any coerciveness assumption on the operator.

Our main result is Theorem 3.1 in Section 3. It is an existence result for a class of nonlinear elliptic problems whose model is the problem (1.1). In the model case, such a theorem states that at least a renormalized solution exists if one of the following conditions holds true:

1) $\gamma=p-1, c \in L^{\frac{N}{p-1}, r}(\Omega), r<+\infty$, and $\|c\|_{L^{\frac{N}{p-1}, r}(\Omega)}$ is small enough;

2) $\gamma<p-1$ and $c \in L^{\frac{N}{p-1}, \infty}(\Omega)$.

The proof of such a result is obtained in various steps. The first difficulty is to obtain some a priori estimate for $|\nabla u|^{p-1}$. By adapting a technique used in G2 (cf. [B]), this is done by decomposing $|\nabla u|^{p-1}$ in two terms

$$
\begin{aligned}
|\nabla u|^{p-1} & =\chi_{\left\{|u| \leq m_{1}\right\}}|\nabla u|^{p-1}+\chi_{\left\{|u|>m_{1}\right\}}|\nabla u|^{p-1} \\
& =\left|\nabla T_{m_{1}}(u)\right|^{p-1}+\left|\nabla S_{m_{1}}(u)\right|^{p-1},
\end{aligned}
$$

where $S_{m_{1}}(u)=u-T_{m_{1}}(u)$ is the "remainder" of the truncation $T_{m_{1}}(u)$ and $m_{1}$ is a value suitably chosen. We first prove an a priori estimate for $\left|\nabla S_{m_{1}}(u)\right|^{p-1}$; in this step we use a generalization, proved in [BMMP3], of a result of [BBGGPV], which says that if $v$ is a function such that $T_{k}(v) \in W_{0}^{1, p}(\Omega)$ and if $\left\|\nabla T_{k}(v)\right\|_{\left(L^{p}(\Omega)\right)^{N}}^{p} \leq$ $k M+L$, for every $k>0$, then $\left\||\nabla v|^{p-1}\right\|_{L^{N^{\prime}}, \infty(\Omega)} \leq c$, where $c$ depends on $M, L$ and $\Omega$. Then we prove that $m_{1}$ is uniformly bounded by a constant which depends only on the data $c, b, \mu$ and $\Omega$ and this gives the desired a priori estimate of $|\nabla u|^{p-1}$. Finally we use a stability result, proved in GM], for equations whose prototype is (1.1) with $b=0$, which is an extension of the stability result proved in [DMOP] (see also $\mathrm{MP}$ ). We also recall that in GM we prove the counterpart of Theorem 3.1 , that is, we prove the existence of a renormalized solution when $\mu$ is a Radon measure with bounded variation on $\Omega, \gamma=\lambda=p-1,\|c\|_{L^{\frac{N}{p-1}, r}(\Omega)}, r<+\infty$, is large and $\|b\|_{L^{N, 1}(\Omega)}$ is small enough. It is worth noting that the method used in the present paper to obtain the a priori estimates seems to not allow dealing with the case $\|c\|_{L^{\frac{N}{p-1}, r}(\Omega)}$ large $(r<+\infty)$ and $\|b\|_{L^{N, 1}(\Omega)}$ small enough while it seems that the one performed in $[\mathrm{GM}]$ is not suitable to the case $\|b\|_{L^{N, 1}(\Omega)}$ large and $\|c\|_{L^{\frac{N}{p-1}, r}(\Omega)}$ small $(r<\infty)$. We explicitly remark that the results proved in 
the present paper and those proved in GM imply the existence of a renormalized solution to the model problem (1.1) under the assumption that the norm of the coefficient $c$ or the norm of the coefficient $b$ is small enough.

\section{Notation AND DEFinition OF RENORMALIZED SOLUTION}

2.1. Notation and definitions. In this section we recall some well-known results about the decomposition of measures (cf. [DMOP]) and a few properties of Lorentz spaces.

Let $\Omega$ be a bounded open subset of $\mathbb{R}^{N}, N \geq 2$. Let $p$ and $p^{\prime}$ be real numbers such that $1<p<N$ and $p^{\prime}$ is the Hölder conjugate exponent of $p$, i.e. $1 / p+1 / p^{\prime}=1$.

We denote by $M_{b}(\Omega)$ the space of Radon measures on $\Omega$ with bounded total variation and by $C_{b}^{0}(\Omega)$ the space of bounded, continuous functions on $\Omega$. Moreover $\mu^{+}$and $\mu^{-}$denote the positive and the negative parts of the measure $\mu$, respectively. We say that a sequence $\left\{\mu_{\varepsilon}\right\}$ of measures in $M_{b}(\Omega)$ converges in the narrow topology to a measure $\mu$ in $M_{b}(\Omega)$ if

$$
\lim _{\varepsilon \rightarrow 0} \int_{\Omega} \varphi d \mu_{\varepsilon}=\int_{\Omega} \varphi d \mu
$$

for every $\varphi \in C_{b}^{0}(\Omega)$.

We denote by $\operatorname{cap}_{p}(B, \Omega)$ the standard capacity defined from $W_{0}^{1, p}(\Omega)$ of a Borel set $B$ and we define $M_{0}(\Omega)$ as the set of the measures $\mu$ in $M_{b}(\Omega)$ which are absolutely continuous with respect to the $p$-capacity, i.e. which satisfy $\mu(B)=0$ for every Borel set $B \subseteq \Omega$ such that $\operatorname{cap}_{p}(B, \Omega)=0$. We define $M_{s}(\Omega)$ as the set of all the measures $\mu$ in $M_{b}(\Omega)$ which are singular with respect to the $p$-capacity, i.e. which are concentrated in a set $E \subset \Omega$ such that $\operatorname{cap}_{p}(E, \Omega)=0$.

The following result allows us to split every measure in $M_{b}(\Omega)$ with respect to the $p$-capacity ([FST], Lemma 2.1, and [BGO], Theorem 2.1).

Proposition 2.1. For every measure $\mu$ in $M_{b}(\Omega)$ there exists a unique pair of measures $\left(\mu_{0}, \mu_{s}\right)$, with $\mu_{0} \in M_{0}(\Omega)$ and $\mu_{s} \in M_{s}(\Omega)$, such that $\mu=\mu_{0}+\mu_{s}$. Moreover for any $\mu_{0}$ that belongs to $M_{0}(\Omega)$, there exist $f$ in $L^{1}(\Omega)$ and $g$ in $\left(L^{p^{\prime}}(\Omega)\right)^{N}$ such that

$$
\mu_{0}=f-\operatorname{div}(g)
$$

in the sense of distributions.

The measures $\mu_{0}$ and $\mu_{s}$ will be called the absolutely continuous part and the singular part of $\mu$ with respect to the $p$-capacity.

We also recall that every function $v \in W_{0}^{1, p}(\Omega)$ is measurable with respect to $\mu_{0}$ and belongs to $L^{\infty}\left(\Omega, \mu_{0}\right)$. If $v$ further belongs to $L^{\infty}(\Omega)$, one has

$$
\int_{\Omega} v d \mu_{0}=\int_{\Omega} f v+\int_{\Omega} g \nabla v, \quad \forall v \in W_{0}^{1, p}(\Omega) \cap L^{\infty}(\Omega)
$$

(see, e.g., DMOP, Proposition 2.7).

Combining the previous result and the Hahn decomposition theorem, we get the following result.

Proposition 2.2. Every measure $\mu$ in $M_{b}(\Omega)$ can be decomposed as follows:

$$
\mu=\mu_{0}+\mu_{s}=f-\operatorname{div}(g)+\mu_{s}^{+}-\mu_{s}^{-},
$$


where $\mu_{0}$ is a measure in $M_{0}(\Omega)$, hence can be written as $f-\operatorname{div}(g)$, with $f \in L^{1}(\Omega)$ and $g \in\left(L^{p^{\prime}}(\Omega)\right)^{N}$, and where $\mu_{s}^{+}$and $\mu_{s}^{-}$(the positive and the negative parts of $\left.\mu_{s}\right)$ are two nonnegative measures in $M_{s}(\Omega)$, which are concentrated on two disjoint subsets $E^{+}$and $E^{-}$of zero p-capacity.

We recall now the definition and a few properties of Lorentz spaces, which we will use in the following. For references about Lorentz spaces see, for example, Lo, O.

Let us denote by $f^{*}$ the decreasing rearrangement of $f$, i.e. the decreasing function defined by

$$
f^{*}(t)=\inf \{s \geq 0: \text { meas }\{x \in \Omega:|f(x)|>s\}<t\}, \quad t \in[0,|\Omega|] .
$$

For references about rearrangements see, for example, $\mathrm{CR}, \mathrm{K}$.

Moreover for $1<q<\infty$ and $1<s \leq \infty$, denote

$$
\|f\|_{L^{q, s}(\Omega)}= \begin{cases}\left(\int_{0}^{|\Omega|}\left[f^{*}(t) t^{\frac{1}{q}}\right]^{s} \frac{d t}{t}\right)^{1 / s}, & \text { if } s<\infty, \\ \sup _{t>0} t[\operatorname{meas}\{x \in \Omega:|f(x)|>t\}]^{1 / r}, & \text { if } s=\infty .\end{cases}
$$

The Lorentz space $L^{q, s}(\Omega)$ is the space of Lebesgue measurable functions such that

$$
\|f\|_{L^{q, s}(\Omega)}<+\infty .
$$

They are "intermediate spaces" between the Lebesgue spaces, in the sense that, for every $1<s<r<\infty$, we have

$$
L^{r, 1}(\Omega) \subset L^{r, r}(\Omega)=L^{r}(\Omega) \subset L^{r, \infty}(\Omega) \subset L^{s, 1}(\Omega) .
$$

The space $L^{r, \infty}(\Omega)$ is the dual space of $L^{r^{\prime}, 1}(\Omega)$, where $\frac{1}{r}+\frac{1}{r^{\prime}}=1$, and one has the generalized Hölder inequality

$$
\left\{\begin{array}{l}
\forall f \in L^{r, \infty}(\Omega), \forall g \in L^{r^{\prime}, 1}(\Omega), \\
\int_{\Omega}|f g| \leq\|f\|_{L^{r, \infty}(\Omega)}\|g\|_{L^{r^{\prime}, 1}(\Omega)}
\end{array}\right.
$$

More generally, if $1<p<\infty$ and $1 \leq q \leq \infty$, we get

$$
\left\{\begin{array}{l}
\forall f \in L^{p_{1}, q_{1}}(\Omega), \forall g \in L^{p_{2}, q_{2}}(\Omega), \\
\|f g\|_{L^{p, q}(\Omega)} \leq\|f\|_{L^{p_{1}, q_{1}}(\Omega)}\|g\|_{L^{p_{2}, q_{2}}(\Omega)}, \\
\frac{1}{p}=\frac{1}{p_{1}}+\frac{1}{p_{2}}, \quad \frac{1}{q}=\frac{1}{q_{1}}+\frac{1}{q_{2}} .
\end{array}\right.
$$

Improvements of the classical Sobolev inequalities involving Lorentz spaces are proved, for example, in [ALT. In the present paper we will only use the following generalized Sobolev inequality: there exists a positive constant $S_{N, p}$ depending only on $p$ and $N$ such that

$$
\|v\|_{L^{p^{*}, p}(\Omega)} \leq S_{N, p}\|v\|_{W_{0}^{1, p}(\Omega)},
$$

for every $v \in W_{0}^{1, p}(\Omega)$. 
2.2. Definition of renormalized solution. In the present paper we consider a nonlinear elliptic problem which can formally be written as $(2.7)$ $\begin{cases}-\operatorname{div}(a(x, u, \nabla u)+K(x, u))+H(x, u, \nabla u)+G(x, u)=\mu-\operatorname{div}(F) & \text { in } \Omega, \\ u=0 & \text { on } \partial \Omega .\end{cases}$

Here $a: \Omega \times \mathbb{R} \times \mathbb{R}^{N} \rightarrow \mathbb{R}^{N}$ and $K: \Omega \times \mathbb{R} \rightarrow \mathbb{R}^{N}$ are Carathéodory functions satisfying

$$
\begin{gathered}
a(x, s, \xi) \xi \geq \alpha|\xi|^{p}, \quad \alpha>0, \\
|a(x, s, \xi)| \leq c\left[|\xi|^{p-1}+|s|^{p-1}+a_{0}(x)\right], \quad a_{0}(x) \in L^{p^{\prime}}(\Omega), \quad c>0, \\
\quad(a(x, s, \xi)-a(x, s, \eta), \xi-\eta)>0, \quad \xi \neq \eta, \\
\left\{\begin{array}{l}
|K(x, s)| \leq c_{0}(x)|s|^{\gamma}+c_{1}(x), \\
0 \leq \gamma \leq p-1, \quad c_{0} \in L^{\frac{N}{p-1}, r}(\Omega), \quad \frac{N}{p-1} \leq r \leq+\infty, \quad c_{1} \in L^{p^{\prime}}(\Omega),
\end{array}\right.
\end{gathered}
$$

for almost every $x \in \Omega$ and for every $s \in \mathbb{R}, \xi \in \mathbb{R}^{N}, \eta \in \mathbb{R}^{N}$.

Moreover $H: \Omega \times \mathbb{R} \times \mathbb{R}^{N} \rightarrow \mathbb{R}$ and $G: \Omega \times \mathbb{R} \rightarrow \mathbb{R}$ are Carathéodory functions satisfying

$$
\begin{gathered}
\left\{\begin{array}{l}
|H(x, s, \xi)| \leq b_{0}(x)|\xi|^{\lambda}+b_{1}(x) \\
0 \leq \lambda \leq p-1, \quad b_{0} \in L^{N, 1}(\Omega), \quad b_{1} \in L^{1}(\Omega) \\
G(x, s) s \geq 0
\end{array}\right. \\
\left\{\begin{array}{l}
|G(x, s)| \leq d_{1}(x)|s|^{t}+d_{2}(x) \\
d_{1} \in L^{z^{\prime}, 1}(\Omega), \quad d_{2} \in L^{1}(\Omega)
\end{array}\right.
\end{gathered}
$$

for almost every $x \in \Omega$ and for every $s \in \mathbb{R}$ and $\xi \in \mathbb{R}^{N}$, where

$$
0 \leq t<\frac{N(p-1)}{N-p}, \quad z=\frac{N(p-1)}{N-p} \frac{1}{t} \text { and } \frac{1}{z}+\frac{1}{z^{\prime}}=1 .
$$

Finally $\mu$ is a measure in $M_{b}(\Omega)$ which is decomposed as

$$
\mu=f-\operatorname{div}(g)+\mu_{s}^{+}-\mu_{s}^{-},
$$

according to Proposition 2.2, and

$$
F \in\left(L^{p^{\prime}}(\Omega)\right)^{N} .
$$

Remark 2.3. Observe that by (2.3) , if the functions $c_{0}$ and $b_{0}$ belong to the Lebesgue spaces $L^{t}(\Omega)$ for some $t \geq \frac{N}{p-1}$ and $L^{q}(\Omega)$ for some $q>N$, then the conditions $c_{0} \in L^{\frac{N}{p-1}, r}(\Omega), \frac{N}{p-1} \leq r \leq+\infty$, and $b_{0} \in L^{N, 1}(\Omega)$ (as requested in hypotheses (2.11) and (2.12) $)$ are satisfied.

For $k>0$, denote by $T_{k}: \mathbb{R} \rightarrow \mathbb{R}$ the usual truncation at level $k$, that is,

$$
T_{k}(s)= \begin{cases}s, & |s| \leq k, \\ k \operatorname{sign}(s), & |s|>k .\end{cases}
$$

Consider a measurable function $u: \Omega \rightarrow \overline{\mathbb{R}}$ which is finite almost everywhere and satisfies $T_{k}(u) \in W_{0}^{1, p}(\Omega)$ for every $k>0$. Then there exists (see e.g. [BBGGPV], 
Lemma 2.1) a unique measurable0 function $v: \Omega \rightarrow \overline{\mathbb{R}}^{N}$, finite almost everywhere, such that

$$
\nabla T_{k}(u)=v \chi_{\{|u| \leq k\}} \quad \text { almost everywhere in } \quad \Omega, \quad \forall k>0 .
$$

We define the gradient $\nabla u$ of $u$ as this function $v$, and denote $\nabla u=v$. Note that the previous definition does not coincide with the definition of the distributional gradient. However if $v \in\left(L_{l o c}^{1}(\Omega)\right)^{N}$, then $u \in W_{l o c}^{1,1}(\Omega)$ and $v$ is the distributional gradient of $u$. In contrast there are examples of functions $u \notin L_{\text {loc }}^{1}(\Omega)$ (and thus such that the gradient of $u$ in the distributional sense is not defined) for which the gradient $\nabla u$ is defined in the previous sense (see Remarks 2.10 and 2.11, Lemma 2.12 and Example 2.16 in [DMOP]).

Definition 2.4. We say that a function $u: \Omega \rightarrow \overline{\mathbb{R}}$, measurable on $\Omega$, almost everywhere finite, is a renormalized solution of (2.7) if it satisfies the following conditions:

$$
\begin{gathered}
T_{k}(u) \in W_{0}^{1, p}(\Omega), \quad \forall k>0 ; \\
|u|^{p-1} \in L^{\frac{N}{N-p}, \infty}(\Omega) ; \\
|\nabla u|^{p-1} \quad \text { belongs to } \quad L^{N^{\prime}, \infty}(\Omega),
\end{gathered}
$$

where $\nabla u$ is the gradient introduced in (2.18);

$$
\begin{aligned}
& \lim _{n \rightarrow+\infty} \frac{1}{n} \int_{n<u<2 n} a(x, u, \nabla u) \cdot \nabla u \varphi=\int_{\Omega} \varphi d \mu_{s}^{+}, \\
& \lim _{n \rightarrow+\infty} \frac{1}{n} \int_{-2 n<u<-n} a(x, u, \nabla u) \cdot \nabla u \varphi=\int_{\Omega} \varphi d \mu_{s}^{-},
\end{aligned}
$$

for every $\varphi \in C_{b}^{0}(\Omega)$;

$$
\lim _{n \rightarrow+\infty} \frac{1}{n} \int_{n<|u|<2 n}|K(x, u)||\nabla u|=0
$$

and finally

$$
\begin{aligned}
\int_{\Omega} a( & x, u, \nabla u) \cdot \nabla u h^{\prime}(u) v+\int_{\Omega} a(x, u, \nabla u) \cdot \nabla v h(u) \\
& +\int_{\Omega} K(x, u) \cdot \nabla u h^{\prime}(u) v+\int_{\Omega} K(x, u) \cdot \nabla v h(u) \\
& +\int_{\Omega} H(x, u, \nabla u) h(u) v+\int_{\Omega} G(x, u) h(u) v \\
= & \int_{\Omega} f h(u) v+\int_{\Omega}(g+F) \cdot \nabla u h^{\prime}(u) v+\int_{\Omega}(g+F) \cdot \nabla v h(u),
\end{aligned}
$$

for every $v \in W^{1, p}(\Omega) \cap L^{\infty}(\Omega)$ and every $h \in W^{1, \infty}(\mathbb{R})$ with a compact support in $\mathbb{R}$, which are such that $h(u) v \in W_{0}^{1, p}(\Omega)$.

Remark 2.5. Observe that every term in (2.25) is well defined since $T_{k}(u) \in W_{0}^{1, p}(\Omega)$ for any $k>0$ and $h$ has a compact support. In particular, since there exists $M>0$ (depending on $h$ ) such that $\operatorname{supp}(h) \subset[-M, M]$,

$$
\int_{\Omega} K(x, u) \cdot \nabla u h^{\prime}(u) v=\int_{\Omega} K\left(x, T_{M}(u)\right) \cdot \nabla T_{M}(u) h^{\prime}(u) v .
$$


Therefore such an integral is well defined thanks to the assumptions (2.11) and the facts that $T_{M}(u) \in W_{0}^{1, p}(\Omega)$ and $h^{\prime}$ are bounded.

Remark 2.6. Observe that every renormalized solution $u$ of (2.7) is such that

$$
\begin{gathered}
|a(x, u, \nabla u)| \in L^{N^{\prime}, \infty}(\Omega), \quad|K(x, u)| \in L^{N^{\prime}, r}(\Omega), \quad \frac{N}{p-1} \leq r \leq+\infty, \\
G(x, u) \in L^{1}(\Omega) \quad \text { and } \quad H(x, u, \nabla u) \in L^{1}(\Omega),
\end{gathered}
$$

thanks to the conditions (2.20) and (2.21), and the growth conditions (2.9), (2.11), (2.12) and (2.14) on $a, K, H$ and $G$ respectively.

Observe also that, since $p<N$, then $L^{p^{\prime}}(\Omega) \subset L^{\frac{N}{p-1}, r}(\Omega), \frac{N}{p-1} \leq r \leq+\infty$. Therefore the term $K(x, u)$ does not in general belong to $\left(L^{p^{\prime}}(\Omega)\right)^{N}$ and the term $-\operatorname{div}(K(x, u))$ is not in general an element of the dual space $W^{-1, p^{\prime}}(\Omega)$.

Moreover $u$ is a solution of (2.77) in the distributional sense, that is, $u$ satisfies

$$
\begin{gathered}
\int_{\Omega} a(x, u, \nabla u) \cdot \nabla \phi+\int_{\Omega} K(x, u) \cdot \nabla \phi+\int_{\Omega} H(x, u, \nabla u) \phi+\int_{\Omega} G(x, u) \phi \\
=\int_{\Omega} \phi d \mu+\int_{\Omega} F \cdot \nabla \phi
\end{gathered}
$$

for all $\phi \in C_{0}^{\infty}(\Omega)$.

This result follows from a standard technique, by taking $\phi \in C_{0}^{\infty}(\Omega)$ and $h_{n}$ defined by

$$
h_{n}(s)= \begin{cases}0, & |s|>2 n, \\ \frac{2 n-|s|}{n}, & n<|s| \leq 2 n, \\ 1, & |s| \leq n,\end{cases}
$$

in (2.25), and letting $n$ tend to infinity.

\section{Statement of existence Result}

The main result of the present paper is the following existence result.

Theorem 3.1. Under assumptions (2.8)-(2.17), there exists at least one renormalized solution $u$ of (2.7) if one of the following conditions holds true:

1) $\gamma=p-1, c_{0} \in L^{\frac{N}{p-1}, r}(\Omega), r<+\infty$, and $\left\|c_{0}\right\|_{L^{\frac{N}{p-1}, r}(\Omega)}$ is small enough;

2) $\gamma<p-1$ and $c_{0} \in L^{\frac{N}{p-1}, \infty}(\Omega)$.

Remark 3.2. Observe that we assume that $c_{0}$ belongs to $L^{\frac{N}{p-1}, r}(\Omega), r<+\infty$, under the hypothesis 1), while $c_{0}$ belongs to $L^{\frac{N}{p-1}, \infty}(\Omega)$ under the hypothesis 2$)$. This is due to the fact that we use the stability theorem (Theorem 5.1 of [GM]) in order to prove the existence result. Actually such a theorem holds true under the assumption that $\gamma=p-1$ and $c_{0} \in L^{\frac{N}{p-1}, r}(\Omega), r<+\infty$, or under the assumption that $\gamma<p-1$ and $c_{0} \in L^{\frac{N}{p-1}, \infty}(\Omega)$.

Remark 3.3. The "limit case" where $\gamma=p-1$ and $c_{0}$ belongs to the Lorentz space $L^{\frac{N}{p-1}, \infty}(\Omega)$ is not considered in Theorem 3.1. Actually we could prove an existence result under the assumptions that $\gamma=p-1, c_{0} \in L^{\frac{N}{p-1}, \infty}(\Omega)$ with its norm in such a space small enough and the right-hand side $\mu$ a measure belonging to $M_{0}(\Omega)$ (and not a more general measure). This restriction on the right-hand side in the case 
where $c_{0}$ belongs to $L^{\frac{N}{p-1}, \infty}(\Omega)$ seems due to our method, which uses the stability result proved in GM]. Indeed such a result can be proved for a class of problems of type (2.7) (with $G \equiv H \equiv 0$ ) under the assumptions (2.8)-(2.11), (2.16) and (2.17) with $c_{0} \in L^{\frac{N}{p-1}, \infty}(\Omega), \mu \in M_{0}(\Omega)$, i.e. $\mu=f-\operatorname{div}(g)$ (see Remarks 4.2 and 4.6 in GM] .

We will prove Theorem 3.1 by an approximation process. First the bounded Radon measure $\mu$ can be decomposed as

$$
\mu=f-\operatorname{div}(g)+\mu_{s}^{+}-\mu_{s}^{-},
$$

where $f \in L^{1}(\Omega), g \in\left(L^{p^{\prime}}(\Omega)\right)^{N}$ and $\mu_{s}^{+}$and $\mu_{s}^{-}$(the positive and the negative parts of $\left.\mu_{s}\right)$ are two nonnegative measures in $M_{b}(\Omega)$ which are concentrated on two disjoint subsets $E^{+}$and $E^{-}$of zero $p$-capacity, according to Proposition 2.2 ,

As in [DMOP] (cf. [BMMP3]), we approximate the measure $\mu$ by a sequence $\mu_{\varepsilon}$ defined as

$$
\mu_{\varepsilon}=f_{\varepsilon}-\operatorname{div}(g)+\lambda_{\varepsilon}^{\oplus}-\lambda_{\varepsilon}^{\ominus},
$$

where

$$
\left\{\begin{array}{l}
\lambda_{\varepsilon}^{\oplus} \text { is a sequence of nonnegative functions in } L^{p^{\prime}}(\Omega) \\
\text { that converges to } \mu_{s}^{+} \text {in the narrow topology of measures, }
\end{array}\right.
$$

and

$$
\left\{\begin{array}{l}
\lambda_{\varepsilon}^{\ominus} \text { is a sequence of nonnegative functions in } L^{p^{\prime}}(\Omega) \\
\text { that converges to } \mu_{s}^{-} \text {in the narrow topology of measures. }
\end{array}\right.
$$

Observe that $\mu_{\varepsilon}$ belongs to $W^{-1, p^{\prime}}(\Omega)$.

Let us denote

$$
\begin{aligned}
K_{\varepsilon}(x, s) & =K\left(x, T_{1 / \varepsilon}(s)\right), \\
H_{\varepsilon}(x, s, \xi) & =T_{1 / \varepsilon}(H(x, s, \xi)), \\
G_{\varepsilon}(x, s) & =T_{1 / \varepsilon}(G(x, s)) .
\end{aligned}
$$

Therefore, by assumptions (2.11)-(2.14), we have

$$
\begin{gathered}
\left|K_{\varepsilon}(x, s)\right| \leq|K(x, s)| \leq c_{0}(x)|s|^{\gamma}+c_{1}(x), \\
\left|K_{\varepsilon}(x, s)\right| \leq c_{0}(x) \frac{1}{\varepsilon^{\gamma}}+c_{1}(x), \\
\left|H_{\varepsilon}(x, s, \xi)\right| \leq|H(x, s, \xi)| \leq b_{0}(x)|\xi|^{\lambda}+b_{1}(x), \\
\left|H_{\varepsilon}(x, s, \xi)\right| \leq \frac{1}{\varepsilon} \\
G_{\varepsilon}(x, s) s \geq 0 \\
\left|G_{\varepsilon}(x, s)\right| \leq|G(x, s)| \leq d_{1}(x)|s|^{r}+d_{2}(x), \\
\left|G_{\varepsilon}(x, s)\right| \leq \frac{1}{\varepsilon}
\end{gathered}
$$


Let $u_{\varepsilon} \in W_{0}^{1, p}(\Omega)$ be a weak solution of the following problem:

$\left\{\begin{array}{lr}-\operatorname{div}\left(a\left(x, u_{\varepsilon}, \nabla u_{\varepsilon}\right)+K_{\varepsilon}\left(x, u_{\varepsilon}\right)\right)+H_{\varepsilon}\left(x, u_{\varepsilon}, \nabla u_{\varepsilon}\right)+G_{\varepsilon}\left(x, u_{\varepsilon}\right)=\mu_{\varepsilon}-\operatorname{div}(F) & \text { in } \Omega, \\ u_{\varepsilon}=0 & \text { on } \partial \Omega,\end{array}\right.$

i.e.

$$
\left\{\begin{array}{l}
u_{\varepsilon} \in W_{0}^{1, p}(\Omega), \\
\quad+\int_{\Omega} H_{\varepsilon}\left(x, u_{\varepsilon}, \nabla u_{\varepsilon}\right) v+\int_{\Omega} G_{\varepsilon}\left(x, u_{\varepsilon}\right) v \\
=\int_{\Omega} f_{\varepsilon} v+\int_{\Omega}(g+F) \cdot \nabla v+\int_{\Omega} \lambda_{\varepsilon}^{\oplus} v-\int_{\Omega} \lambda_{\varepsilon}^{\ominus} v, \\
\forall v \in W_{0}^{1, p}(\Omega) .
\end{array}\right.
$$

The existence of a solution $u_{\varepsilon}$ of (3.15) is a well-known result (see e.g. [L, DPo2]).

\section{Proof of Theorem 3.1}

The main difficulty in proving Theorem 3.1 is to obtain an a priori estimate of $\left|\nabla u_{\varepsilon}\right|^{p-1}$ in $L^{N^{\prime}, \infty}(\Omega)$. Let us explain our method in the case where $\gamma=\lambda=p-1$. By adapting a proof used in G2, we decompose $\left|\nabla u_{\varepsilon}\right|^{p-1}$ in two terms

$$
\begin{aligned}
\left|\nabla u_{\varepsilon}\right|^{p-1} & =\chi_{\left\{\left|u_{\varepsilon}\right|>m_{1}\right\}}\left|\nabla u_{\varepsilon}\right|^{p-1}+\chi_{\left\{\left|u_{\varepsilon}\right| \leq m_{1}\right\}}\left|\nabla u_{\varepsilon}\right|^{p-1} \\
& =\left|\nabla S_{m_{1}}\left(u_{\varepsilon}\right)\right|^{p-1}+\left|\nabla T_{m_{1}}\left(u_{\varepsilon}\right)\right|^{p-1}
\end{aligned}
$$

where $S_{m_{1}}\left(u_{\varepsilon}\right)=u_{\varepsilon}-T_{m_{1}}\left(u_{\varepsilon}\right)$ is the "remainder" of the truncation $T_{m_{1}}\left(u_{\varepsilon}\right)$ and $m_{1}$ is a value suitably chosen. Then we first prove an a priori estimate for $\left|\nabla S_{m_{1}}\left(u_{\varepsilon}\right)\right|^{p-1}$, with a bound depending on $m_{1}$ and on the data; in this step we use a slighter generalization, proved in [BMMP3], of a result of [BBGGPV], which we state below.

Lemma 4.1. Assume that $\Omega$ is an open subset of $\mathbb{R}^{N}$ with finite measure and that $1<p<N$. Let $u$ be a measurable function satisfying $T_{k}(u) \in W_{0}^{1, p}(\Omega)$, for every positive $k$, and such that

$$
\int_{\Omega}\left|\nabla T_{k}(u)\right|^{p} \leq M k+L, \quad \forall k>0,
$$

where $M$ and $L$ are given constants. Then $|u|^{p-1}$ belongs to $L^{\frac{p^{*}}{p}, \infty}(\Omega),|\nabla u|^{p-1}$ belongs to $L^{N^{\prime}, \infty}(\Omega)$ and

$$
\begin{gathered}
\left\||u|^{p-1}\right\|_{L^{\frac{p^{*}}{p}, \infty}(\Omega)} \leq C(N, p)\left[M+|\Omega|^{\frac{1}{p^{*}}} L^{\frac{1}{p^{\prime}}}\right], \\
\left\||\nabla u|^{p-1}\right\|_{L^{N^{\prime}, \infty}(\Omega)} \leq C(N, p)\left[M+|\Omega|^{\frac{1}{N^{\prime}}-\frac{1}{p^{\prime}}} L^{\frac{1}{p^{\prime}}}\right],
\end{gathered}
$$

where $C(N, p)$ is a constant depending only on $N$ and $p$ and where $\frac{1}{p^{*}}=\frac{1}{p}-\frac{1}{N}$.

Second, we give an a priori estimate of $\left|\nabla T_{m_{1}}\left(u_{\varepsilon}\right)\right|^{p-1}$ depending on $m_{1}$ and on the data. The third step is devoted to prove that $m_{1}$ is uniformly bounded by a constant which is independent on $\varepsilon$; this allows us to obtain the a priori estimate of $\left|\nabla u_{\varepsilon}\right|^{p-1}$. In the last section we prove that the approximated terms $H_{\varepsilon}\left(x, u_{\varepsilon}, \nabla u_{\varepsilon}\right)$ 
and $G_{\varepsilon}\left(x, u_{\varepsilon}\right)$ converge strongly in $L^{1}(\Omega)$; this allows us to reproduce the proof of the stability result proved in [GM] (Theorem 5.1), which is a slight generalization of the stability result of [DMOP].

4.1. A priori estimates. The main step of the proof of Theorem 3.1 consists of proving an a priori estimate of $\left|\nabla u_{\varepsilon}\right|^{p-1}$ in $L^{N^{\prime}, \infty}(\Omega)$. Let us explicitly remark that such an a priori estimate holds true under more general assumptions on summability of $c_{0}$ of Theorem 3.1 (see Remark 4.3).

Theorem 4.2. Under the assumptions of Theorem 3.1, every solution $u_{\varepsilon}$ of (3.15) satisfies

$$
\begin{aligned}
& \left\|\left|\nabla u_{\varepsilon}\right|^{p-1}\right\|_{L^{N^{\prime}, \infty}(\Omega)} \leq c \\
& \left\|\left|u_{\varepsilon}\right|^{p-1}\right\|_{L^{\frac{N}{N-p}, \infty}(\Omega)} \leq c,
\end{aligned}
$$

where $c$ is a positive constant which depends only on $p,|\Omega|, N, \alpha,\left\|b_{0}\right\|_{L^{N, 1}(\Omega)}$, $\left\|b_{1}\right\|_{L^{1}(\Omega)},\left\|c_{0}\right\|_{L^{\frac{N}{p-1}, r}(\Omega)},\left\|c_{1}\right\|_{L^{p^{\prime}}(\Omega)},\|g\|_{\left(L^{p^{\prime}}(\Omega)\right)^{N}},\|F\|_{\left(L^{p^{\prime}}(\Omega)\right)^{N}}, \sup _{\varepsilon}\left\|f_{\varepsilon}\right\|_{L^{1}(\Omega)}$, $\sup _{\varepsilon}\left(\lambda_{\varepsilon}^{\oplus}(\Omega)+\lambda_{\varepsilon}^{\ominus}(\Omega)\right)$ and on the decreasing rearrangement $\left(b_{0}\right)^{*}$ of $b_{0}$.

Proof. We begin to prove Theorem 4.2 under assumption 1) of Theorem 3.1, i.e. when $\gamma=\lambda=p-1$ and $\left\|c_{0}\right\|_{L^{\frac{N}{p-1}, r}(\Omega)}$ is small enough.

Observe that, since $r<+\infty, L^{\frac{N}{p-1}, r}(\Omega) \subset L^{\frac{N}{p-1}, \infty}(\Omega)$. Therefore $c_{0}$ belongs to $L^{\frac{N}{p-1}, \infty}(\Omega)$ and, moreover, since we assume that $\left\|c_{0}\right\|_{L^{\frac{N}{p-1}, r}(\Omega)}$ is small enough, we also have that $\left\|c_{0}\right\|_{L^{\frac{N}{p-1}}, \infty}(\Omega)$ is small too. From now on we will use that

$$
c_{0} \in L^{\frac{N}{p-1}, \infty}(\Omega) \text { and }\left\|c_{0}\right\|_{L^{\frac{N}{p-1}, \infty}(\Omega)} \text { is small enough. }
$$

As in BMMP3, we define the following set $Z_{\varepsilon}$. As $|\Omega|$ is finite, the set of the constants $c$ such that $\left|\left\{x \in \Omega,\left|u_{\varepsilon}(x)\right|=c\right\}\right|>0$ is at most countable. Let $Z_{\varepsilon}^{c}$ be the (countable) union of all those sets. Its complementary $Z_{\varepsilon}=\Omega-Z_{\varepsilon}^{c}$ is therefore the union of the sets such that $\left|\left\{x \in \Omega,\left|u_{\varepsilon}(x)\right|=c\right\}\right|=0$. Since for every $c$

$$
\nabla u_{\varepsilon}=0 \quad \text { a.e. on }\left\{x \in \Omega,\left|u_{\varepsilon}(x)\right|=c\right\},
$$

and since $Z_{\varepsilon}^{c}$ is at most a countable union, we obtain that

$$
\nabla u_{\varepsilon}=0 \quad \text { a.e. on } Z_{\varepsilon}^{c} \text {. }
$$

First step. Using the techniques developed in BMMP3, we give in this step an estimate on $S_{m_{1}}\left(u_{\varepsilon}\right)$ where $m_{1}$ is a positive real number depending on $\varepsilon$ and on the data.

Define, for $m>0$, the function $S_{m}: \mathbb{R} \rightarrow \mathbb{R}$ by

$$
S_{m}(s)=s-T_{m}(s),
$$

i.e.

$$
S_{m}(s)= \begin{cases}0, & |s| \leq m, \\ (|s|-m) \operatorname{sign}(s), & |s|>m .\end{cases}
$$


We use in (3.15) the test function $T_{k}\left(S_{m}\left(u_{\varepsilon}\right)\right)$ and we obtain

$$
\begin{aligned}
\int_{\Omega} a & \left(x, u_{\varepsilon}, \nabla u_{\varepsilon}\right) \cdot \nabla T_{k}\left(S_{m}\left(u_{\varepsilon}\right)\right)+\int_{\Omega} K_{\varepsilon}\left(x, u_{\varepsilon}\right) \cdot \nabla T_{k}\left(S_{m}\left(u_{\varepsilon}\right)\right) \\
& +\int_{\Omega} H_{\varepsilon}\left(x, u_{\varepsilon}, \nabla u_{\varepsilon}\right) T_{k}\left(S_{m}\left(u_{\varepsilon}\right)\right)+\int_{\Omega} G_{\varepsilon}\left(x, u_{\varepsilon}\right) T_{k}\left(S_{m}\left(u_{\varepsilon}\right)\right) \\
= & \int_{\Omega} f_{\varepsilon} T_{k}\left(S_{m}\left(u_{\varepsilon}\right)\right)+\int_{\Omega}(g+F) \cdot \nabla T_{k}\left(S_{m}\left(u_{\varepsilon}\right)\right) \\
& +\int_{\Omega} \lambda_{\varepsilon}^{\oplus} T_{k}\left(S_{m}\left(u_{\varepsilon}\right)\right)-\int_{\Omega} \lambda_{\varepsilon}^{\ominus} T_{k}\left(S_{m}\left(u_{\varepsilon}\right)\right) .
\end{aligned}
$$

Now we estimate the various terms in (4.9).

By the definition (4.8) of $S_{m}(s)$ and the ellipticity condition (2.8), we obtain

$$
\begin{aligned}
\int_{\Omega} a\left(x, u_{\varepsilon}, \nabla u_{\varepsilon}\right) \cdot \nabla T_{k}\left(S_{m}\left(u_{\varepsilon}\right)\right) & =\int_{\left\{m \leq\left|u_{\varepsilon}\right| \leq m+k\right\}} a\left(x, u_{\varepsilon}, \nabla u_{\varepsilon}\right) \cdot \nabla u_{\varepsilon} \\
& \geq \alpha \int_{\Omega}\left|\nabla T_{k}\left(S_{m}\left(u_{\varepsilon}\right)\right)\right|^{p} .
\end{aligned}
$$

Let us now estimate $\left|\int_{\Omega} K_{\varepsilon}\left(x, u_{\varepsilon}\right) \cdot \nabla T_{k}\left(S_{m}\left(u_{\varepsilon}\right)\right)\right|$.

Let

$$
\beta_{p}=\max \left\{1,2^{p-1}\right\} .
$$

By the definition (4.8) of $S_{m}(s)$, the growth condition (3.7) on $K_{\varepsilon}$, the generalized Sobolev inequality (2.6), the generalized Hölder inequality (2.4) and the Young inequality, we get

$$
\begin{aligned}
&\left|\int_{\Omega} K_{\varepsilon}\left(x, u_{\varepsilon}\right) \cdot \nabla T_{k}\left(S_{m}\left(u_{\varepsilon}\right)\right)\right| \\
& \leq \int_{\Omega} c_{0}\left|u_{\varepsilon}\right|^{p-1}\left|\nabla T_{k}\left(S_{m}\left(u_{\varepsilon}\right)\right)\right|+\int_{\Omega} c_{1}\left|\nabla T_{k}\left(S_{m}\left(u_{\varepsilon}\right)\right)\right| \\
& \leq \beta_{p} \int_{\Omega} c_{0}\left(\left|u_{\varepsilon}\right|-m\right)^{p-1}\left|\nabla T_{k}\left(S_{m}\left(u_{\varepsilon}\right)\right)\right|+\beta_{p} m^{p-1} \int_{\Omega} c_{0}\left|\nabla T_{k}\left(S_{m}\left(u_{\varepsilon}\right)\right)\right| \\
&+\int_{\Omega} c_{1}\left|\nabla T_{k}\left(S_{m}\left(u_{\varepsilon}\right)\right)\right| \\
& \leq \beta_{p}\left\|c_{0}\right\|_{L^{\frac{N}{p-1}, \infty}(\Omega)}\left\|T_{k}\left(S_{m}\left(u_{\varepsilon}\right)\right)\right\|_{L^{p^{*}, p}(\Omega)}^{p-1}\left\|\nabla T_{k}\left(S_{m}\left(u_{\varepsilon}\right)\right)\right\|_{\left(L^{p}(\Omega)\right)^{N}} \\
&+\beta_{p} m^{p-1}\|1\|_{L^{\frac{p^{*}}{p-1}}, p^{\prime}}(\Omega) \\
&+\left\|c_{0}\right\|_{L^{\frac{N}{p-1}, \infty}(\Omega)}\left\|\nabla T_{k}\left(S_{m}\left(u_{\varepsilon}\right)\right)\right\|_{\left(L^{p}(\Omega)\right)^{N}} \\
& \leq\left.\beta_{p^{p^{\prime}}(\Omega)} S_{N, p}\left\|c_{0}\right\|_{L^{\frac{N}{p-1}, \infty}(\Omega)}\left\|\nabla T_{k}\left(S_{m}\left(u_{\varepsilon}\right)\right)\right\|_{\left(L^{p}(\Omega)\right)^{N}}\left(u_{\varepsilon}\right)\right) \|_{\left(L^{p}(\Omega)\right)^{N}}^{p} \\
&+\frac{\beta_{p}^{p^{\prime}}}{p^{\prime}}\|1\|_{L^{\frac{p^{*}}{p-1}, p^{\prime}}(\Omega)}^{p^{\prime}}\left\|c_{0}\right\|_{L^{\frac{N}{p-1}, \infty}(\Omega)} m^{p}+\frac{1}{p}\left\|c_{0}\right\|_{L^{\frac{N}{p-1}, \infty}(\Omega)}\left\|\nabla T_{k}\left(S_{m}\left(u_{\varepsilon}\right)\right)\right\|_{\left(L^{p}(\Omega)\right)^{N}}^{p} \\
&+\frac{2^{p^{\prime} / p}}{p^{\prime} \alpha^{p^{\prime} / p}}\left\|c_{1}\right\|_{L^{p^{\prime}}(\Omega)}^{p^{\prime}}+\frac{\alpha}{2 p}\left\|\nabla T_{k}\left(S_{m}\left(u_{\varepsilon}\right)\right)\right\|_{\left(L^{p}(\Omega)\right)^{N}}^{p}
\end{aligned}
$$




$$
\begin{aligned}
= & \left(\beta_{p} S_{N, p}+\frac{1}{p}\right)\left\|c_{0}\right\|_{L^{\frac{N}{p-1}, \infty}(\Omega)}\left\|\nabla T_{k}\left(S_{m}\left(u_{\varepsilon}\right)\right)\right\|_{\left(L^{p}(\Omega)\right)^{N}}^{p} \\
& +\frac{\alpha}{2 p}\left\|\nabla T_{k}\left(S_{m}\left(u_{\varepsilon}\right)\right)\right\|_{\left(L^{p}(\Omega)\right)^{N}}^{p} \\
& +\frac{\beta_{p}^{p^{\prime}}}{p^{\prime}}\|1\|_{L^{\frac{p^{*}}{p-1}, p^{\prime}}(\Omega)}^{p^{\prime}}\left\|c_{0}\right\|_{L^{\frac{N}{p-1}, \infty}(\Omega)} m^{p}+\frac{2^{p^{\prime} / p}}{p^{\prime} \alpha^{p^{\prime} / p}}\left\|c_{1}\right\|_{L^{p^{\prime}}(\Omega)}^{p^{\prime}} .
\end{aligned}
$$

Let us now estimate $\left|\int_{\Omega} H_{\varepsilon}\left(x, u_{\varepsilon}, \nabla u_{\varepsilon}\right) T_{k}\left(S_{m}\left(u_{\varepsilon}\right)\right)\right|$.

By the definition (4.8) of $S_{m}$, the growth assumption (3.9) on $H_{\varepsilon}$ and the generalized Hölder inequality (2.4), we have

$$
\begin{aligned}
\mid \int_{\Omega} H_{\varepsilon}\left(x, u_{\varepsilon},\right. & \left.\nabla u_{\varepsilon}\right) T_{k}\left(S_{m}\left(u_{\varepsilon}\right)\right) \mid \\
& \leq k \int_{\left\{\left|u_{\varepsilon}\right|>m\right\}}\left|H_{\varepsilon}\left(x, u_{\varepsilon}, \nabla u_{\varepsilon}\right)\right| \\
& \leq k\left[\int_{\left\{\left|u_{\varepsilon}\right|>m\right\}} b_{0}\left|\nabla u_{\varepsilon}\right|^{p-1}+\int_{\Omega} b_{1}\right] \\
& =k\left[\int_{Z_{\varepsilon} \cap\left\{\left|u_{\varepsilon}\right|>m\right\}} b_{0}\left|\nabla S_{m}\left(u_{\varepsilon}\right)\right|^{p-1}+\int_{\Omega} b_{1}\right] \\
& \leq k\left[\left\|b_{0}\right\|_{L^{N, 1}\left(Z_{\varepsilon} \cap\left\{u_{\varepsilon}>m\right\}\right)}\left\|\left|\nabla S_{m}\left(u_{\varepsilon}\right)\right|^{p-1}\right\|_{L^{N^{\prime}, \infty}(\Omega)}+\left\|b_{1}\right\|_{L^{1}(\Omega)}\right] .
\end{aligned}
$$

Moreover, by the "sign condition" (3.11) on $G_{\varepsilon}$, we get

$$
\int_{\Omega} G_{\varepsilon}\left(x, u_{\varepsilon}\right) T_{k}\left(S_{m}\left(u_{\varepsilon}\right)\right) \geq 0 .
$$

Finally

$\int_{\Omega}(g+F) \cdot \nabla T_{k}\left(S_{m}\left(u_{\varepsilon}\right)\right) \leq \frac{\alpha}{2 p}\left\|\nabla T_{k}\left(S_{m}\left(u_{\varepsilon}\right)\right)\right\|_{\left(L^{p}(\Omega)\right)^{N}}^{p}+\frac{2^{p^{\prime} / p}}{p^{\prime} \alpha^{p^{\prime} / p}}\|g+F\|_{\left(L^{p^{\prime}}(\Omega)\right)^{N}}^{p^{\prime}}$,

$$
\begin{aligned}
& \left|\int_{\Omega} \lambda_{\varepsilon}^{\oplus} T_{k}\left(S_{m}\left(u_{\varepsilon}\right)\right)\right| \leq k \lambda_{\varepsilon}^{\oplus}(\Omega), \\
& \left|\int_{\Omega} \lambda_{\varepsilon}^{\ominus} T_{k}\left(S_{m}\left(u_{\varepsilon}\right)\right)\right| \leq k \lambda_{\varepsilon}^{\ominus}(\Omega) .
\end{aligned}
$$

Denote

$$
C_{1}=\frac{\alpha}{p^{\prime}}-\left(\beta_{p} S_{N, p}+\frac{1}{p}\right)\left\|c_{0}\right\|_{L^{\frac{N}{p-1}, \infty}(\Omega)} .
$$

Observe that, since $\left\|c_{0}\right\|_{L^{\frac{N}{p-1}}, \infty}(\Omega)$ is small enough, from now on we can assume

$$
\left\|c_{0}\right\|_{L^{\frac{N}{p-1}, \infty}(\Omega)}<\frac{p \alpha}{p^{\prime}\left(\beta_{p} p S_{N, p}+1\right)},
$$

so that $C_{1}$ is a positive constant. 
Combining (4.9)-(4.17), we get

$$
\left\|\nabla T_{k}\left(S_{m}\left(u_{\varepsilon}\right)\right)\right\|_{\left(L^{p}(\Omega)\right)^{N}}^{p} \leq M k+L, \quad \forall k>0,
$$

where $M$ and $L$ are defined by

$$
\begin{aligned}
& \left\{\begin{array}{l}
M=\frac{1}{C_{1}}\left(\left\|b_{0}\right\|_{L^{N, 1}\left(Z_{\varepsilon} \cap\left\{\left|u_{\varepsilon}\right|>m\right\}\right)}\left\|\left|\nabla S_{m}\left(u_{\varepsilon}\right)\right|^{p-1}\right\|_{L^{N^{\prime}, \infty}(\Omega)}+M_{0}\right), \\
M_{0}=\left\|b_{1}\right\|_{L^{1}(\Omega)}+\sup _{\varepsilon}\left\|f_{\varepsilon}\right\|_{L^{1}(\Omega)}+\sup _{\varepsilon}\left[\lambda_{\varepsilon}^{\oplus}(\Omega)+\lambda_{\varepsilon}^{\ominus}(\Omega)\right], \\
L_{1}=\frac{1}{C_{1}} \frac{\beta_{p}^{p^{\prime}}}{p^{\prime}}\|1\|_{L^{\frac{p^{*}}{p-1} p^{\prime}(\Omega)}}^{p^{\prime}}\left\|c_{0}\right\|_{L^{\frac{N}{p-1}, \infty}(\Omega)}, \\
L_{0}=\frac{1}{C_{1}} \frac{2^{p^{\prime} / p}}{p^{\prime} \alpha^{p^{\prime} / p}}\left(\left\|c_{1}\right\|_{L^{p^{\prime}(\Omega)}}^{p^{\prime}}+\|g+F\|_{\left(L^{p^{\prime}}(\Omega)\right)^{N}}^{p^{\prime}}\right) .
\end{array}\right.
\end{aligned}
$$

By Lemma 4.1, we get

$$
\begin{aligned}
&\left\|\left|\nabla S_{m}\left(u_{\varepsilon}\right)\right|^{p-1}\right\|_{L^{N^{\prime}}, \infty}(\Omega) \\
& \leq C(N, p)\left[M+|\Omega|^{\frac{1}{N^{\prime}}-\frac{1}{p^{\prime}}} L^{\frac{1}{p^{\prime}}}\right] \\
& \leq C^{\prime}(N, p)\left[\frac{1}{C_{1}}\left\|b_{0}\right\|_{L^{N, 1}\left(Z_{\varepsilon} \cap\left\{\left|u_{\varepsilon}\right|>m\right\}\right)}\left\|\left|\nabla S_{m}\left(u_{\varepsilon}\right)\right|^{p-1}\right\|_{L^{N^{\prime}}, \infty}(\Omega)\right. \\
&\left.+\frac{M_{0}}{C_{1}}+|\Omega|^{\frac{1}{N^{\prime}}-\frac{1}{p^{\prime}}} L_{1}^{\frac{1}{p^{\prime}}} m^{p-1}+|\Omega|^{\frac{1}{N^{\prime}}}-\frac{1}{p^{\prime}} L_{0}^{\frac{1}{p^{\prime}}}\right] .
\end{aligned}
$$

Denote by $\left(b_{0_{Z_{\varepsilon} \cap\left\{\left|u_{\varepsilon}\right|>m\right\}}}\right)^{*}$ and $\left(b_{0}\right)^{*}$ the decreasing rearrangements of the restriction $b_{0_{Z_{\varepsilon} \cap\left\{\left|u_{\varepsilon}\right|>m\right\}}}$ and of $b_{0}$, respectively.

By the definition (2.1) of norm of Lorentz spaces and the definition of the decreasing rearrangement, it is easy to verify that the following inequality holds true:

$$
\begin{aligned}
\left\|b_{0}\right\|_{L^{N, 1}\left(Z_{\varepsilon} \cap\left\{\left|u_{\varepsilon}\right|>m\right\}\right)} & =\int_{0}^{\left|Z_{\varepsilon} \cap\left\{\left|u_{\varepsilon}\right|>m\right\}\right|}\left(b_{0_{Z_{\varepsilon} \cap\left\{\left|u_{\varepsilon}\right|>m\right\}}}\right)^{*}(t) t^{\frac{1}{N}} \frac{d t}{t} \\
& \leq \int_{0}^{\left|Z_{\varepsilon} \cap\left\{\left|u_{\varepsilon}\right|>m\right\}\right|}\left(b_{0}\right)^{*}(t) t^{1 / N} \frac{d t}{t} .
\end{aligned}
$$

In the case where

$$
\frac{C(N, p)}{C_{1}}\left\|b_{0}\right\|_{L^{N, 1}\left(Z_{\varepsilon}\right)}=\frac{C(N, p)}{C_{1}} \int_{0}^{\left|Z_{\varepsilon}\right|}\left(b_{0}\right)^{*}(t) t^{1 / N} \frac{d t}{t} \leq \frac{1}{2},
$$

we choose $m=m_{1}=0$ and the proof is complete. Let us assume that (4.25) does not hold. Since the function $m \rightarrow\left|Z_{\varepsilon} \cap\left\{\left|u_{\varepsilon}\right|>m\right\}\right|$ is continuous (indeed the constants $c$, such that the sets $\left\{\left|u_{\varepsilon}(x)\right|=c\right\}$ have a strictly positive measure, have been eliminated by considering $Z_{\varepsilon}$ ), decreasing and tends to 0 when $m$ tends to $\infty$, we can choose $m=m_{1}>0$ such that

$$
\frac{C(N, p)}{C_{1}} \int_{0}^{\left|Z_{\varepsilon} \cap\left\{\left|u_{\varepsilon}\right|>m_{1}\right\}\right|}\left(b_{0}\right)^{*}(t) t^{1 / N} \frac{d t}{t}=\frac{1}{2} .
$$


Moreover, we define $\delta$ by

$$
\frac{C(N, p)}{C_{1}} \int_{0}^{\delta}\left(b_{0}\right)^{*}(t) t^{1 / N} \frac{d t}{t}=\frac{1}{2} .
$$

Then we have

$$
\left|Z_{\varepsilon} \cap\left\{\left|u_{\varepsilon}\right|>m_{1}\right\}\right|=\delta .
$$

Observe that $\delta$ does not depend on $\varepsilon$.

Choosing $m=m_{1}$, we obtain from 4.23)

$$
\left\|\left|\nabla S_{m_{1}}\left(u_{\varepsilon}\right)\right|^{p-1}\right\|_{L^{N^{\prime}, \infty}(\Omega)} \leq 2 C(N, p)\left[\frac{M_{0}}{C_{1}}+|\Omega|^{\frac{1}{N^{\prime}}-\frac{1}{p^{\prime}}} L_{1}^{\frac{1}{p^{\prime}}} m_{1}^{p-1}+|\Omega|^{\frac{1}{N^{\prime}}-\frac{1}{p^{\prime}}} L_{0}^{\frac{1}{p^{\prime}}}\right],
$$

where $M_{0}, L_{0}$ and $L_{1}$ are defined by (4.21) and (4.22).

Second step. We now give an estimate on $T_{m_{1}}\left(u_{\varepsilon}\right)$.

Using the test function $T_{m_{1}}\left(u_{\varepsilon}\right)$ in (3.15), we obtain

$$
\begin{aligned}
\int_{\Omega} a & \left(x, u_{\varepsilon}, \nabla u_{\varepsilon}\right) \cdot \nabla T_{m_{1}}\left(u_{\varepsilon}\right)+\int_{\Omega} K_{\varepsilon}\left(x, u_{\varepsilon}\right) \cdot \nabla T_{m_{1}}\left(u_{\varepsilon}\right) \\
& +\int_{\Omega} H_{\varepsilon}\left(x, u_{\varepsilon}, \nabla u_{\varepsilon}\right) T_{m_{1}}\left(u_{\varepsilon}\right)+\int_{\Omega} G_{\varepsilon}\left(x, u_{\varepsilon}\right) T_{m_{1}}\left(u_{\varepsilon}\right) \\
= & \int_{\Omega} f_{\varepsilon} T_{m_{1}}\left(u_{\varepsilon}\right)+\int_{\Omega}(g+F) \cdot \nabla T_{m_{1}}\left(u_{\varepsilon}\right) \\
& +\int_{\Omega} \lambda_{\varepsilon}^{\oplus} T_{m_{1}}\left(u_{\varepsilon}\right)-\int_{\Omega} \lambda_{\varepsilon}^{\ominus} T_{m_{1}}\left(u_{\varepsilon}\right) .
\end{aligned}
$$

Now we evaluate the various terms in 4.29).

By the ellipticity condition (2.8), we obtain

$$
\begin{aligned}
\int_{\Omega} a\left(x, u_{\varepsilon}, \nabla u_{\varepsilon}\right) \cdot \nabla T_{m_{1}}\left(u_{\varepsilon}\right) & =\int_{\left\{\left|u_{\varepsilon}\right| \leq m_{1}\right\}} a\left(x, u_{\varepsilon}, \nabla u_{\varepsilon}\right) \cdot \nabla u_{\varepsilon} \\
& \geq \alpha \int_{\Omega}\left|\nabla T_{m_{1}}\left(u_{\varepsilon}\right)\right|^{p} .
\end{aligned}
$$

Let us now estimate $\left|\int_{\Omega} K_{\varepsilon}\left(x, u_{\varepsilon}\right) \cdot \nabla T_{m_{1}}\left(u_{\varepsilon}\right)\right|$.

By the growth condition (3.7) on $K_{\varepsilon}$, the generalized Sobolev inequality (2.6), the generalized Hölder inequality (2.4) and the Young inequality, we get

$$
\begin{aligned}
&\left|\int_{\Omega} K_{\varepsilon}\left(x, u_{\varepsilon}\right) \cdot \nabla T_{m_{1}}\left(u_{\varepsilon}\right)\right| \\
& \leq \int_{\Omega} c_{0}\left|u_{\varepsilon}\right|^{p-1}\left|\nabla T_{m_{1}}\left(u_{\varepsilon}\right)\right|+\int_{\Omega} c_{1}\left|\nabla T_{m_{1}}\left(u_{\varepsilon}\right)\right| \\
& \leq\left\|c_{0}\right\|_{L^{\frac{N}{p-1}, \infty}(\Omega)}\left\|T_{m_{1}}\left(u_{\varepsilon}\right)\right\|_{L^{p^{*}, p}(\Omega)}^{p-1}\left\|\nabla T_{m_{1}}\left(u_{\varepsilon}\right)\right\|_{\left(L^{p}(\Omega)\right)^{N}} \\
&+\left\|c_{1}\right\|_{L^{p^{\prime}}(\Omega)}\left\|\nabla T_{m_{1}}\left(u_{\varepsilon}\right)\right\|_{\left(L^{p}(\Omega)\right)^{N}} \\
& \leq S_{N, p}\left\|c_{0}\right\|_{L^{\frac{N}{p-1}, \infty}(\Omega)}\left\|\nabla T_{m_{1}}\left(u_{\varepsilon}\right)\right\|_{\left(L^{p}(\Omega)\right)^{N}}^{p} \\
&+\frac{4^{p^{\prime} / p}}{p^{\prime} \alpha^{p^{\prime} / p}}\left\|c_{1}\right\|_{L^{p^{\prime}(\Omega)}}^{p^{\prime}}+\frac{\alpha}{4 p}\left\|\nabla T_{m_{1}}\left(u_{\varepsilon}\right)\right\|_{\left(L^{p}(\Omega)\right)^{N} .}^{p}
\end{aligned}
$$


Let us now estimate $\left|\int_{\Omega} H_{\varepsilon}\left(x, u_{\varepsilon}, \nabla u_{\varepsilon}\right) T_{m_{1}}\left(u_{\varepsilon}\right)\right|$.

By the growth assumption (3.9) on $H_{\varepsilon}$ and the generalized Hölder inequality (2.4), we have

$$
\begin{aligned}
\mid \int_{\Omega} H_{\varepsilon}\left(x, u_{\varepsilon},\right. & \left.\nabla u_{\varepsilon}\right) T_{m_{1}}\left(u_{\varepsilon}\right) \mid \\
\leq & m_{1} \int_{\Omega}\left|H_{\varepsilon}\left(x, u_{\varepsilon}, \nabla u_{\varepsilon}\right)\right| \\
\leq & m_{1}\left[\int_{\left\{\left|u_{\varepsilon}\right| \leq m_{1}\right\}} b_{0}\left|\nabla u_{\varepsilon}\right|^{p-1}+\int_{\left\{\left|u_{\varepsilon}\right|>m_{1}\right\}} b_{0}\left|\nabla u_{\varepsilon}\right|^{p-1}+\int_{\Omega} b_{1}\right] \\
\leq & \frac{2^{p / p^{\prime}} m_{1}^{p}}{p \alpha^{p / p^{\prime}}}\left\|b_{0}\right\|_{L^{p}(\Omega)}^{p}+\frac{\alpha}{2 p^{\prime}}\left\|\nabla T_{m_{1}}\left(u_{\varepsilon}\right)\right\|_{\left(L^{p}(\Omega)\right)^{N}}^{p} \\
& +m_{1}\left[\left\|b_{0}\right\|_{L^{N, 1}\left(Z_{\varepsilon} \cap\left\{u_{\varepsilon}>m_{1}\right\}\right)}\left\|\left|\nabla S_{m_{1}}\left(u_{\varepsilon}\right)\right|^{p-1}\right\|_{L^{N^{\prime}, \infty}(\Omega)}+\left\|b_{1}\right\|_{L^{1}(\Omega)}\right] .
\end{aligned}
$$

Moreover, by the "sign condition" (3.11) on $G_{\varepsilon}$, we get

$$
\int_{\Omega} G_{\varepsilon}\left(x, u_{\varepsilon}\right) T_{m_{1}}\left(u_{\varepsilon}\right) \geq 0
$$

Finally we have

$$
\int_{\Omega} f_{\varepsilon} T_{m_{1}}\left(u_{\varepsilon}\right) \leq m_{1}\left\|f_{\varepsilon}\right\|_{L^{1}(\Omega)}
$$

$$
\begin{aligned}
\int_{\Omega}(g+F) \cdot \nabla T_{m_{1}}\left(u_{\varepsilon}\right) \leq & \frac{\alpha}{4 p}\left\|\nabla T_{m_{1}}\left(u_{\varepsilon}\right)\right\|_{\left(L^{p}(\Omega)\right)^{N}}^{p}+\frac{4^{p^{\prime} / p}}{p^{\prime} \alpha^{p^{\prime} / p}}\|g+F\|_{\left(L^{p^{\prime}}(\Omega)\right)^{N}}^{p^{\prime}}, \\
& \left|\int_{\Omega} \lambda_{\varepsilon}^{\oplus} T_{m_{1}}\left(u_{\varepsilon}\right)\right| \leq m_{1} \lambda_{\varepsilon}^{\oplus}(\Omega), \\
& \left|\int_{\Omega} \lambda_{\varepsilon}^{\ominus} T_{m_{1}}\left(u_{\varepsilon}\right)\right| \leq m_{1} \lambda_{\varepsilon}^{\ominus}(\Omega) .
\end{aligned}
$$

Denote

$$
C_{2}=\frac{\alpha}{2}-S_{N, p}\left\|c_{0}\right\|_{L^{\frac{N}{p-1}, \infty}(\Omega)} .
$$

Since $\left\|c_{0}\right\|_{L^{\frac{N}{p-1}}, \infty}(\Omega)$ is small enough, from now on we can suppose

$$
\left\|c_{0}\right\|_{L^{\frac{N}{p-1}, \infty}(\Omega)}<\frac{\alpha}{2 S_{N, p}},
$$

so that $C_{2}$ is a positive constant (recall that the norm of $c_{0}$ also satisfies (4.19)). 
Combining (4.29)-(4.37), we get

$$
\begin{aligned}
\| \nabla & T_{m_{1}}\left(u_{\varepsilon}\right) \|_{\left(L^{p}(\Omega)\right)^{N}}^{p} \\
\leq & \frac{1}{C_{2}}\left\{\frac{2^{p / p^{\prime}} m_{1}^{p}}{p \alpha^{p / p^{\prime}}}\left\|b_{0}\right\|_{L^{p}(\Omega)}^{p}\right. \\
& +m_{1}\left[\left\|b_{0}\right\|_{L^{N, 1}\left(Z_{\varepsilon} \cap\left\{u_{\varepsilon}>m_{1}\right\}\right)}\left\|\left|\nabla S_{m_{1}}\left(u_{\varepsilon}\right)\right|^{p-1}\right\|_{L^{N^{\prime}, \infty}(\Omega)}+M_{0}\right] \\
& \left.+\frac{4^{p^{\prime} / p}}{p^{\prime} \alpha^{p^{\prime} / p}}\left(\left\|c_{1}\right\|_{L^{p^{\prime}(\Omega)}}^{p^{\prime}}+\|g+F\|_{\left(L^{p^{\prime}}(\Omega)\right)^{N}}^{p^{\prime}}\right)\right\},
\end{aligned}
$$

where $M_{0}$ is defined by (4.21).

Third step. In this step we prove that $m_{1}$ is uniformly bounded with respect to $\varepsilon$. It is performed through a technical "log-type" estimate on $u_{\varepsilon}$ (cf. [B], G2]).

To this end, let us define for $h>0$ the function $\phi_{h}: \mathbb{R} \rightarrow \mathbb{R}$ by

$$
\phi_{h}(s)=\left\{\frac{1}{\left[(h+1) m_{1}-\left|T_{m_{1}}(s)\right|\right]^{p-1}}-\frac{1}{\left[(h+1) m_{1}\right]^{p-1}}\right\} \operatorname{sign}(s),
$$

i.e.

$$
\phi_{h}(s)= \begin{cases}{\left[\frac{1}{\left((h+1) m_{1}-|s|\right)^{p-1}}-\frac{1}{\left[(h+1) m_{1}\right]^{p-1}}\right] \operatorname{sign}(s),} & |s| \leq m_{1}, \\ {\left[\frac{1}{\left(h m_{1}\right)^{p-1}}-\frac{1}{\left((h+1) m_{1}\right)^{p-1}}\right] \operatorname{sign}(s),} & |s|>m_{1} .\end{cases}
$$

Observe that the following property of $\phi_{h}(s)$ holds true:

$$
\left|\phi_{h}(s)\right| \leq \frac{1}{\left(h m_{1}\right)^{p-1}}, \quad \forall s \in \mathbb{R} .
$$

Since $\phi_{h}(s)$ is a Lipschitz continuous function with $\phi_{h}(0)=0$ and since $u_{\varepsilon} \in$ $W_{0}^{1, p}(\Omega)$, the function $\phi_{h}\left(u_{\varepsilon}\right)$ belongs to $W_{0}^{1, p}(\Omega)$. This allows us to use $\phi_{h}\left(u_{\varepsilon}\right)$ as a test function in (3.15). Then we get

$$
\begin{aligned}
\int_{\Omega} a\left(x, u_{\varepsilon}, \nabla\right. & \left.u_{\varepsilon}\right) \cdot \nabla u_{\varepsilon} \phi_{h}^{\prime}\left(u_{\varepsilon}\right)+\int_{\Omega} K_{\varepsilon}\left(x, u_{\varepsilon}\right) \cdot \nabla u_{\varepsilon} \phi_{h}^{\prime}\left(u_{\varepsilon}\right) \\
& +\int_{\Omega} H_{\varepsilon}\left(x, u_{\varepsilon}, \nabla u_{\varepsilon}\right) \phi_{h}\left(u_{\varepsilon}\right)+\int_{\Omega} G_{\varepsilon}\left(x, u_{\varepsilon}\right) \phi_{h}\left(u_{\varepsilon}\right) \\
= & \int_{\Omega} f_{\varepsilon} \phi_{h}\left(u_{\varepsilon}\right)+\int_{\Omega}(g+F) \cdot \nabla u_{\varepsilon} \phi_{h}^{\prime}\left(u_{\varepsilon}\right)+\int_{\Omega} \lambda_{\varepsilon}^{\oplus} \phi_{h}\left(u_{\varepsilon}\right)-\int_{\Omega} \lambda_{\varepsilon}^{\ominus} \phi_{h}\left(u_{\varepsilon}\right) .
\end{aligned}
$$

Now we estimate the various integrals in (4.43).

By the definition (4.41) of $\phi_{h}(s)$ and the ellipticity condition (2.8), we obtain

$$
\begin{aligned}
\int_{\Omega} a\left(x, u_{\varepsilon}, \nabla u_{\varepsilon}\right) \cdot \nabla u_{\varepsilon} \phi_{h}^{\prime}\left(u_{\varepsilon}\right) & \geq \int_{\left\{\left|u_{\varepsilon}\right| \leq m_{1}\right\}} a\left(x, u_{\varepsilon}, \nabla u_{\varepsilon}\right) \cdot \nabla u_{\varepsilon} \phi_{h}^{\prime}\left(u_{\varepsilon}\right) \\
& \geq(p-1) \alpha \int_{\Omega} \frac{\left|\nabla T_{m_{1}}\left(u_{\varepsilon}\right)\right|^{p}}{\left[(h+1) m_{1}-\left|T_{m_{1}}\left(u_{\varepsilon}\right)\right|\right]^{p}} .
\end{aligned}
$$


Let us now estimate $\left|\int_{\Omega} K_{\varepsilon}\left(x, u_{\varepsilon}\right) \cdot \phi_{h}^{\prime}\left(u_{\varepsilon}\right) \nabla u_{\varepsilon}\right|$.

Since, $m_{1}-\left|T_{m_{1}}(s)\right| \geq 0$ for any $s \in \mathbb{R}$, the growth condition (3.7) on $K_{\varepsilon}$ and the Young inequality yield

$$
\begin{aligned}
& \int_{\Omega} K_{\varepsilon}\left(x, u_{\varepsilon}\right) \cdot \nabla u_{\varepsilon} \phi_{h}^{\prime}\left(u_{\varepsilon}\right) \\
& \leq \int_{\Omega} c_{0}\left|u_{\varepsilon}\right|^{p-1}\left|\nabla u_{\varepsilon}\right|\left|\phi_{h}^{\prime}\left(u_{\varepsilon}\right)\right|+\int_{\Omega} c_{1}\left|\nabla u_{\varepsilon}\right|\left|\phi_{h}^{\prime}\left(u_{\varepsilon}\right)\right| \\
& =(p-1) \int_{\left|u_{\varepsilon}\right| \leq m_{1}} \frac{c_{0}\left|u_{\varepsilon}\right|^{p-1}\left|\nabla u_{\varepsilon}\right|}{\left[(h+1) m_{1}-\left|u_{\varepsilon}\right|\right]^{p}}+(p-1) \int_{\left|u_{\varepsilon}\right| \leq m_{1}} \frac{c_{1}\left|\nabla u_{\varepsilon}\right|}{\left[(h+1) m_{1}-\left|u_{\varepsilon}\right|\right]^{p}} \\
& \leq \frac{3^{p^{\prime} / p}(p-1)}{p^{\prime} \alpha^{p^{\prime} / p}} \int_{\Omega} \frac{c_{0}^{p^{\prime}} m_{1}^{p}}{\left[(h+1) m_{1}-\left|T_{m_{1}}\left(u_{\varepsilon}\right)\right|\right]^{p}}+\frac{(p-1) \alpha}{3 p} \int_{\Omega} \frac{\left|\nabla u_{\varepsilon}\right|^{p}}{\left[(h+1) m_{1}-\left|T_{m_{1}}\left(u_{\varepsilon}\right)\right|\right]^{p}} \\
& \quad+\frac{3^{p^{\prime} / p}(p-1)}{p^{\prime} \alpha^{p^{\prime} / p}} \int_{\Omega} \frac{c_{1}^{p^{\prime}}}{\left[(h+1) m_{1}-\left|T_{m_{1}}\left(u_{\varepsilon}\right)\right|\right]^{p}}+\frac{(p-1) \alpha}{3 p} \int_{\Omega} \frac{\left|\nabla u_{\varepsilon}\right|^{p}}{\left[(h+1) m_{1}-\left|T_{m_{1}}\left(u_{\varepsilon}\right)\right|\right]^{p}} \\
& \leq \frac{2(p-1) \alpha}{3 p} \int_{\Omega} \frac{\left.\left|(h+1) m_{1}-\right| T_{m_{1}}\left(u_{\varepsilon}\right) \mid\right]^{p}}{[(h+p} \\
& \quad+\frac{3^{p^{\prime} / p}(p-1)}{p^{\prime} \alpha^{p^{\prime} / p}}\left(\frac{1}{h^{p}}\left\|c_{0}\right\|_{L^{p^{\prime}}(\Omega)}^{p^{\prime}}+\frac{1}{\left(h m_{1}\right)^{p}}\left\|c_{1}\right\|_{L^{p^{\prime}}(\Omega)}^{p^{\prime}}\right) .
\end{aligned}
$$

Moreover, since $p<N$, we have $L^{\frac{N}{p-1}, \infty}(\Omega) \subset L^{p^{\prime}}(\Omega)$ and by inequality (2.5) it follows that

$$
\left\|c_{0}\right\|_{L^{p^{\prime}}(\Omega)} \leq\|1\|_{L^{\frac{p N}{(p-1)(N-p)}, p^{\prime}}(\Omega)}\left\|c_{0}\right\|_{L^{\frac{N}{p-1}, \infty}(\Omega)}
$$

i.e.

$$
\left\|c_{0}\right\|_{L^{p^{\prime}}(\Omega)} \leq \frac{N}{N-p}|\Omega|^{\frac{N-p}{N}}\left\|c_{0}\right\|_{L^{\frac{N}{p-1}, \infty}(\Omega)}
$$

Therefore, we obtain

$$
\begin{aligned}
& \int_{\Omega} K_{\varepsilon}\left(x, u_{\varepsilon}\right) \cdot \nabla u_{\varepsilon} \phi_{h}^{\prime}\left(u_{\varepsilon}\right) \mid \\
& \leq \frac{2(p-1) \alpha}{3 p} \int_{\Omega} \frac{\left|\nabla u_{\varepsilon}\right|^{p}}{\left[(h+1) m_{1}-\left|T_{m_{1}}\left(u_{\varepsilon}\right)\right|\right]^{p}} \\
& \quad+\frac{3^{p^{\prime} / p}(p-1)}{p^{\prime} \alpha^{p^{\prime} / p}}\left[\frac{1}{h^{p}}\left(\frac{N}{N-p}\right)^{p^{\prime}}|\Omega|^{\frac{(N-p) p^{\prime}}{N}}\left\|c_{0}\right\|_{L^{\frac{N}{p-1}, \infty}(\Omega)}^{p^{\prime}}+\frac{1}{\left(h m_{1}\right)^{p}}\left\|c_{1}\right\|_{L^{p^{\prime}}(\Omega)}^{p^{\prime}}\right]
\end{aligned}
$$

Let us now estimate $\left|\int_{\Omega} H_{\varepsilon}\left(x, u_{\varepsilon}, \nabla u_{\varepsilon}\right) \phi_{h}\left(u_{\varepsilon}\right)\right|$. 
By the definition (4.41) of $\phi_{h}$, the growth assumption (3.9) on $H_{\varepsilon}$, the property (4.42) and the generalized Hölder inequality (2.4), we have

$$
\begin{aligned}
\mid \int_{\Omega} & H_{\varepsilon}\left(x, u_{\varepsilon}, \nabla u_{\varepsilon}\right) \phi_{h}\left(u_{\varepsilon}\right) \mid \\
\leq & \int_{\Omega} b_{0}\left|\nabla u_{\varepsilon}\right|^{p-1}\left|\phi_{h}\left(u_{\varepsilon}\right)\right|+\int_{\Omega} b_{1}\left|\phi_{h}\left(u_{\varepsilon}\right)\right| \\
\leq & \int_{Z_{\varepsilon} \cap\left\{\left|u_{\varepsilon}\right| \leq m_{1}\right\}} \frac{b_{0}\left|\nabla u_{\varepsilon}\right|^{p-1}}{\left[(h+1) m_{1}-\left|u_{\varepsilon}\right|\right]^{p-1}} \\
& +\frac{1}{\left(h m_{1}\right)^{p-1}} \int_{Z_{\varepsilon} \cap\left\{\left|u_{\varepsilon}\right|>m_{1}\right\}} b_{0}\left|\nabla u_{\varepsilon}\right|^{p-1}+\frac{1}{\left(h m_{1}\right)^{p-1}}\left\|b_{1}\right\|_{L^{1}(\Omega)} \\
\leq & \frac{2^{p / p^{\prime}}}{[p(p-1) \alpha]^{p / p^{\prime}}}\left\|b_{0}\right\|_{L^{p}(\Omega)}^{p}+\frac{(p-1) \alpha}{2 p^{\prime}} \int_{\left|u_{\varepsilon}\right| \leq m_{1}} \frac{\left|\nabla u_{\varepsilon}\right|^{p}}{\left[(h+1) m_{1}-\left|T_{m_{1}}\left(u_{\varepsilon}\right)\right|\right]^{p}} \\
& +\frac{1}{\left(h m_{1}\right)^{p-1}}\left[\left\|b_{0}\right\|_{L^{N, 1}\left(Z_{\varepsilon} \cap\left\{u_{\varepsilon}>m\right\}\right)}\left\|\left|\nabla S_{m_{1}}\left(u_{\varepsilon}\right)\right|^{p-1}\right\|_{L^{N^{\prime}, \infty}(\Omega)}+\left\|b_{1}\right\|_{L^{1}(\Omega)}\right] .
\end{aligned}
$$

Since $p<N$, we have $L^{N, 1}(\Omega) \subset L^{p}(\Omega)$ and therefore the coefficient $b_{0}$ belongs to $L^{p}(\Omega)$.

Moreover, by the "sign condition" (3.11) of $G_{\varepsilon}$, we get

$$
\int_{\Omega} G_{\varepsilon}\left(x, u_{\varepsilon}\right) \phi_{h}\left(u_{\varepsilon}\right) \geq 0 .
$$

Finally, since for any $s \in \mathbb{R}$ we have $(h+1) m_{1}-\left|T_{m_{1}}(s)\right| \geq h m_{1}$, we get

$$
\begin{aligned}
& \int_{\Omega}(g+F) \cdot \nabla u_{\varepsilon} \phi_{h}^{\prime}\left(u_{\varepsilon}\right)=(p-1) \int_{\left|u_{\varepsilon}\right| \leq m_{1}} \frac{(g+F) \cdot \nabla u_{\varepsilon}}{\left[(h+1) m_{1}-\left|u_{\varepsilon}\right|\right]^{p}} \\
& \quad \leq \frac{3^{p^{\prime} / p}(p-1)}{p^{\prime} \alpha^{p^{\prime} / p}\left(h m_{1}\right)^{p}}\|g+F\|_{\left(L^{\left.p^{\prime}(\Omega)\right)^{N}}\right.}^{p^{\prime}}+\frac{(p-1) \alpha}{3 p} \int_{\Omega} \frac{\left|\nabla u_{\varepsilon}\right|^{p}}{\left[(h+1) m_{1}-\left|T_{m_{1}}\left(u_{\varepsilon}\right)\right|\right]^{p}}
\end{aligned}
$$

and, the property (4.42) of $\phi_{h}$ gives that

$$
\begin{aligned}
& \int_{\Omega} f_{\varepsilon} \phi_{h}\left(u_{\varepsilon}\right) \leq \frac{1}{\left(h m_{1}\right)^{p-1}}\left\|f_{\varepsilon}\right\|_{L^{1}(\Omega)}, \\
& \left|\int_{\Omega} \lambda_{\varepsilon}^{\oplus} \phi_{h}\left(u_{\varepsilon}\right)\right| \leq \frac{1}{\left(h m_{1}\right)^{p-1}} \lambda_{\varepsilon}^{\oplus}(\Omega), \\
& \left|\int_{\Omega} \lambda_{\varepsilon}^{\ominus} \phi_{h}\left(u_{\varepsilon}\right)\right| \leq \frac{1}{\left(h m_{1}\right)^{p-1}} \lambda_{\varepsilon}^{\ominus}(\Omega) .
\end{aligned}
$$

Gathering (4.43)-4.51) leads to

$$
\begin{aligned}
& \int_{\Omega} \frac{\left|\nabla T_{m_{1}}\left(u_{\varepsilon}\right)\right|^{p}}{\left[(h+1) m_{1}-\left.\left|T_{m_{1}}\left(u_{\varepsilon}\right)\right|\right|^{p}\right.} \\
& \quad \leq C(p, N,|\Omega|, \alpha)\left\{\left\|b_{0}\right\|_{L^{p}(\Omega)}^{p}+\frac{1}{\left(h m_{1}\right)^{p-1}}\left\|b_{0}\right\|_{L^{N, 1}\left(Z_{\varepsilon} \cap\left\{u_{\varepsilon}>m_{1}\right\}\right)}\left\|\left.\nabla S_{m_{1}}\left(u_{\varepsilon}\right)\right|^{p-1}\right\|_{L^{N^{\prime}, \infty}(\Omega)}\right. \\
& \left.\quad+\frac{1}{\left(h m_{1}\right)^{p-1}} M_{0}+\frac{1}{\left(h m_{1}\right)^{p}}\left(\left\|c_{1}\right\|_{L^{p^{\prime}}(\Omega)}^{p^{\prime}}+\|g+F\|_{\left(L^{p^{\prime}}(\Omega)\right)^{N}}^{p^{\prime}}\right)+\frac{1}{h^{p}}\left\|c_{0}\right\|_{L^{\frac{N}{p-1}, \infty}}^{p^{\prime}}\right\}
\end{aligned}
$$


662

OLIVIER GUIBÉ AND ANNA MERCALDO

where $M_{0}$ is defined by (4.21) and where

$$
\begin{aligned}
C(p, N,|\Omega|, \alpha) & =\frac{2 p^{\prime}}{(p-1) \alpha} \max \left\{\frac{2^{p / p^{\prime}}}{[p(p-1) \alpha]^{p / p^{\prime}}}, 1,\right. \\
& \left.\frac{3^{p^{\prime} / p}(p-1)}{p^{\prime} \alpha^{p^{\prime} / p}}, \frac{3^{p^{\prime} / p}(p-1)}{p^{\prime} \alpha^{p^{\prime} / p}}\left(\frac{N}{N-p}\right)^{p^{\prime}}|\Omega| \frac{(N-p) p^{\prime}}{N}\right\} .
\end{aligned}
$$

On the one hand, using the estimate (4.28) of $\left|\nabla S_{m_{1}}\left(u_{\varepsilon}\right)\right|^{p-1}$ in the first step, together with the Young inequality and the definition (4.22) of $L_{1}$ yields that

$$
\begin{aligned}
& \int_{\Omega} \frac{\left|\nabla T_{m_{1}}\left(u_{\varepsilon}\right)\right|^{p}}{\left[(h+1) m_{1}-\left|T_{m_{1}}\left(u_{\varepsilon}\right)\right|\right]^{p}} \\
& \leq C^{\prime}(p, N,|\Omega|, \alpha)\left\{\left\|b_{0}\right\|_{L^{p}(\Omega)}^{p}+\frac{1}{\left(h m_{1}\right)^{p-1}}\left\|b_{0}\right\|_{L^{N, 1}\left(Z_{\varepsilon} \cap\left\{u_{\varepsilon}>m_{1}\right\}\right)}\right. \\
& \times\left[M_{0}+L_{1}^{\frac{1}{p^{\prime}}} m_{1}^{p-1}+L_{0}^{\frac{1}{p^{\prime}}}\right] \\
& \left.+\frac{1}{\left(h m_{1}\right)^{p-1}} M_{0}+\frac{1}{\left(h m_{1}\right)^{p}}\left(\left\|c_{1}\right\|_{L^{p^{\prime}(\Omega)}}^{p^{\prime}}+\|g+F\|_{\left(L^{p^{\prime}}(\Omega)\right)^{N}}^{p^{\prime}}\right)+\frac{1}{h^{p}}\left\|c_{0}\right\|_{L^{\frac{N}{p-1}, \infty}(\Omega)}^{p^{\prime}}\right\} \\
& \leq C^{\prime}(p, N,|\Omega|, \alpha)\left\{\left\|b_{0}\right\|_{L^{p}(\Omega)}^{p}+\frac{1}{h^{p-1}}\left\|b_{0}\right\|_{L^{N, 1}\left(Z_{\varepsilon} \cap\left\{u_{\varepsilon}>m_{1}\right\}\right)} L_{1}^{\frac{1}{p^{\prime}}}\right. \\
& +\frac{1}{\left(h m_{1}\right)^{p-1}}\left[\left\|b_{0}\right\|_{L^{N, 1}(\Omega)}\left(M_{0}+L_{0}^{\frac{1}{p^{\prime}}}\right)+M_{0}\right] \\
& \left.+\frac{1}{\left(h m_{1}\right)^{p}}\left(\left\|c_{1}\right\|_{L^{p^{\prime}(\Omega)}}^{p^{\prime}}+\|g+F\|_{\left(L^{p^{\prime}}(\Omega)\right)^{N}}^{p^{\prime}}\right)+\frac{1}{h^{p}}\left\|c_{0}\right\|_{L^{\frac{N}{p-1}, \infty}(\Omega)}^{p^{\prime}}\right\} \\
& \leq C^{\prime}(p, N,|\Omega|, \alpha)\left\{\left\|b_{0}\right\|_{L^{p}(\Omega)}^{p}+\frac{1}{p}\left\|b_{0}\right\|_{L^{N, 1}(\Omega)}^{p}+\frac{1}{p^{\prime} h^{p}} L_{1}\right. \\
& +\frac{1}{\left(h m_{1}\right)^{p-1}}\left[\left\|b_{0}\right\|_{L^{N, 1}(\Omega)}\left(M_{0}+L_{0}^{\frac{1}{p}}\right)+M_{0}\right] \\
& \left.+\frac{1}{\left(h m_{1}\right)^{p}}\left(\left\|c_{1}\right\|_{L^{p^{\prime}}(\Omega)}^{p^{\prime}}+\|g+F\|_{\left(L^{p^{\prime}}(\Omega)\right)^{N}}^{p^{\prime}}\right)+\frac{1}{h^{p}}\left\|c_{0}\right\|_{L^{\frac{N}{p-1} \infty}(\Omega)}^{p^{\prime}}\right\}, \\
& \leq C^{\prime}(p, N,|\Omega|, \alpha)\left\{\left\|b_{0}\right\|_{L^{p}(\Omega)}^{p}\right. \\
& +\frac{1}{p}\left\|b_{0}\right\|_{L^{N, 1}(\Omega)}^{p}+\frac{1}{\left(h m_{1}\right)^{p-1}}\left[\left\|b_{0}\right\|_{L^{N, 1}(\Omega)}\left(M_{0}+L_{0}^{\frac{1}{p^{\prime}}}\right)+M_{0}\right] \\
& +\frac{1}{\left(h m_{1}\right)^{p}}\left(\left\|c_{1}\right\|_{L^{p^{\prime}}(\Omega)}^{p^{\prime}}+\|g+F\|_{\left(L^{p^{\prime}}(\Omega)\right)^{N}}^{p^{\prime}}\right) \\
& \left.+\frac{1}{h^{p}}\left(\left\|c_{0}\right\|_{L^{\frac{N}{p-1}, \infty}(\Omega)}^{p^{\prime}}+\left\|c_{0}\right\|_{L^{\frac{N}{p-1}, \infty}(\Omega)}\right)\right\} \text {, }
\end{aligned}
$$

where $L_{0}$ and $L_{1}$ are defined by (4.22) and where

$$
C^{\prime}(p, N,|\Omega|, \alpha)=C(p, N,|\Omega|, \alpha) \max \left\{1,2 C(N, p)\left(\frac{\beta_{p}^{p^{\prime}}}{C_{1} p^{\prime 2}}\|1\|_{L^{\frac{p^{*}}{p-1}, p^{\prime}}}+1\right)\right\} .
$$


On the other hand, due to (4.27), we have

$$
\operatorname{meas}\left(Z_{\varepsilon} \cap\left\{u_{\varepsilon}>m_{1}\right\}\right)=\delta \leq \operatorname{meas}\left(\left\{u_{\varepsilon}>m_{1}\right\}\right),
$$

and by the Poincaré inequality, we get

$$
\begin{aligned}
& \int_{\Omega} \frac{\left|\nabla T_{m_{1}}\left(u_{\varepsilon}\right)\right|^{p}}{\left[(h+1) m_{1}-\left|T_{m_{1}}\left(u_{\varepsilon}\right)\right|\right]^{p}}=\int_{\Omega}\left|\nabla \log \left[(h+1)\left(m_{1}+1\right)-\left|T_{m_{1}}\left(u_{\varepsilon}\right)\right|\right]\right|^{p} \\
& \quad=\int_{\Omega}\left|\nabla \log \left[1-\frac{\left|T_{m_{1}}\left(u_{\varepsilon}\right)\right|}{(h+1) m_{1}}\right]\right|^{p} \\
& \quad \geq c(|\Omega|, p) \int_{\Omega}\left|\log \left[1-\frac{\left|T_{m_{1}}\left(u_{\varepsilon}\right)\right|}{(h+1) m_{1}}\right]\right|^{p} \\
& \quad \geq c(|\Omega|, p) \int_{\left|u_{\varepsilon}\right|>m_{1}}\left|\log \left[1-\frac{\left|T_{m_{1}}\left(u_{\varepsilon}\right)\right|}{(h+1) m_{1}}\right]\right|^{p} \\
& \quad \geq c(|\Omega|, p)\left|\log \left[1-\frac{m_{1}}{(h+1) m_{1}}\right]\right|^{p} \delta \\
& \quad=c(|\Omega|, p)\left[\log \left(1+\frac{1}{h}\right)\right]^{p} \delta .
\end{aligned}
$$

Combining (4.52) and (4.53), we obtain

$$
\begin{aligned}
{\left[\log \left(1+\frac{1}{h}\right)\right]^{p} \leq } & \frac{C^{\prime \prime}(p, N,|\Omega|, \alpha)}{\delta}\left\{\left\|b_{0}\right\|_{L^{p}(\Omega)}^{p}+\frac{1}{p}\left\|b_{0}\right\|_{L^{N, 1}(\Omega)}^{p}\right. \\
& +\frac{1}{\left(h m_{1}\right)^{p-1}}\left[\left\|b_{0}\right\|_{L^{N, 1}(\Omega)}\left(M_{0}+L_{0}^{\frac{1}{p^{\prime}}}\right)+M_{0}\right] \\
& +\frac{1}{\left(h m_{1}\right)^{p}}\left(\left\|c_{1}\right\|_{L^{p^{\prime}}(\Omega)}^{p^{\prime}}+\|g+F\|_{\left(L^{p^{\prime}}(\Omega)\right)^{N}}^{p^{\prime}}\right) \\
& \left.+\frac{1}{h^{p}}\left(\left\|c_{0}\right\|_{L^{\frac{N}{p-1}, \infty}(\Omega)}^{p^{\prime}}+\left\|c_{0}\right\|_{L^{\frac{N}{p-1}, \infty}(\Omega)}\right)\right\}
\end{aligned}
$$

We are now in a position to prove that $m_{1}$ is uniformly bounded with respect to $\varepsilon$ by a suitable choice of $h$ in (4.54) and if $\left\|c_{0}\right\|_{L^{\frac{N}{p-1}, \infty}(\Omega)}$ is small enough. We first fix $h=h_{1}$ such that

$$
\frac{C^{\prime \prime}(p, N,|\Omega|, \alpha)}{\delta}\left(\left\|b_{0}\right\|_{L^{p}(\Omega)}^{p}+\frac{1}{p}\left\|b_{0}\right\|_{L^{N, 1}(\Omega)}^{p}\right)=\frac{1}{2}\left[\log \left(1+\frac{1}{h_{1}}\right)\right]^{p} .
$$

Observe that $h_{1}$ is independent on $\varepsilon$. Therefore we get from (4.54)

$$
\begin{aligned}
& {\left[\log \left(1+\frac{1}{h}\right)\right]^{p}} \\
& \leq \frac{2 C^{\prime \prime}(p, N,|\Omega|, \alpha)}{\delta}\left\{\frac{1}{\left(h m_{1}\right)^{p-1}}\left[\left\|b_{0}\right\|_{L^{N, 1}(\Omega)}\left(M_{0}+L_{0}^{\frac{1}{p^{\prime}}}\right)+M_{0}\right]\right. \\
& \quad+\frac{1}{\left(h m_{1}\right)^{p}}\left(\left\|c_{1}\right\|_{L^{p^{\prime}}(\Omega)}^{p^{\prime}}+\|g+F\|_{\left(L^{p^{\prime}}(\Omega)\right)^{N}}^{p^{\prime}}\right) \\
& \left.\quad+\frac{1}{h^{p}}\left(\left\|c_{0}\right\|_{L^{\frac{N}{p-1}, \infty}(\Omega)}^{p^{\prime}}+\left\|c_{0}\right\|_{L^{\frac{N}{p-1}, \infty}(\Omega)}\right)\right\} .
\end{aligned}
$$


Denote

$$
\begin{gathered}
a_{1}=\left[\log \left(1+\frac{1}{h_{1}}\right)\right]^{p}-\frac{2 C^{\prime \prime}(p, N,|\Omega|, \alpha)}{\delta h_{1}^{p}}\left\|c_{0}\right\|_{L^{\frac{N}{p-1}, \infty}(\Omega)}^{p^{\prime}}, \\
a_{2}=\frac{2 C^{\prime \prime}(p, N,|\Omega|, \alpha)}{\delta h_{1}^{p-1}}\left[\left\|b_{0}\right\|_{L^{N, 1}(\Omega)}\left(M_{0}+L_{0}^{\frac{1}{p^{\prime}}}\right)+M_{0}\right], \\
a_{3}=\frac{2 C^{\prime \prime}(p, N,|\Omega|, \alpha)}{\delta h_{1}^{p}}\left(\left\|c_{1}\right\|_{L^{p^{\prime}}(\Omega)}^{p^{\prime}}+\|g+F\|_{\left(L^{p^{\prime}}(\Omega)\right)^{N}}^{p^{\prime}}\right) .
\end{gathered}
$$

Since $\left\|c_{0}\right\|_{L^{\frac{N}{p-1}, \infty}(\Omega)}$ is small enough, we can assume

$$
\frac{2 C^{\prime \prime}(p, N,|\Omega|, \alpha)}{\delta h_{1}^{p}}\left(\left\|c_{0}\right\|_{L^{\frac{N}{p-1}, \infty}(\Omega)}^{p^{\prime}}+\left\|c_{0}\right\|_{L^{\frac{N}{p-1}, \infty}(\Omega)}\right)<\left[\log \left(1+\frac{1}{h_{1}}\right)\right]^{p},
$$

so that $a_{1}$ is a positive constant (recall that the norm of $c_{0}$ also satisfies (4.19) and (4.39)).

Observe also that $a_{1}, a_{2}$ and $a_{3}$ are constants independent on $\varepsilon$.

Therefore by (4.55) we get

$$
a_{1}<\frac{a_{2}}{m_{1}^{p-1}}+\frac{a_{3}}{m_{1}^{p}}
$$

which allows to conclude that

$$
m_{1} \leq c
$$

where $c$ is a constant which does not depend on $\varepsilon .^{1}$

By the estimate (4.28) of $\left|\nabla S_{m_{1}}\left(u_{\varepsilon}\right)\right|^{p-1}$ in the first step, we deduce that

$$
\left\|\left|\nabla S_{m_{1}}\left(u_{\varepsilon}\right)\right|^{p-1}\right\|_{L^{p}(\Omega)}^{p} \leq c,
$$

and therefore by the estimate (4.40) of $\nabla T_{m_{1}}\left(u_{\varepsilon}\right)$ in the second step, we also get

$$
\left\|\nabla T_{m_{1}}\left(u_{\varepsilon}\right)\right\|_{\left(L^{p}(\Omega)\right)^{N}}^{p} \leq c .
$$

Moreover, writing

$$
\begin{aligned}
\left|\nabla u_{\varepsilon}\right|^{p-1} & =\left|\nabla u_{\varepsilon}\right|^{p-1} \chi_{\left\{\left|u_{\varepsilon}\right| \leq m_{1}\right\}}+\left|\nabla u_{\varepsilon}\right|^{p-1} \chi_{\left\{\left|u_{\varepsilon}\right|>m_{1}\right\}} \\
& =\left|\nabla T_{m_{1}}\left(u_{\varepsilon}\right)\right|^{p-1}+\left|\nabla S_{m_{1}}\left(u_{\varepsilon}\right)\right|^{p-1}
\end{aligned}
$$

and using (4.57) and (4.58) lead to

$$
\begin{aligned}
\left\|\left|\nabla u_{\varepsilon}\right|^{p-1}\right\|_{L^{N^{\prime}, \infty}(\Omega)} & \left.\leq\left\|\left|\nabla T_{m_{1}}\left(u_{\varepsilon}\right)\right|^{p-1}\right\|_{\left(L^{N^{\prime}}, \infty\right.}(\Omega)\right)^{N}+\left\|\left|\nabla S_{m_{1}}\left(u_{\varepsilon}\right)\right|^{p-1}\right\|_{L^{N^{\prime}}, \infty}(\Omega) \\
& \leq c\left\|\nabla T_{m_{1}}\left(u_{\varepsilon}\right)\right\|_{\left(L^{p}(\Omega)\right)^{N}}+\left\|\left.\nabla S_{m_{1}}\left(u_{\varepsilon}\right)\right|^{p-1}\right\|_{L^{N^{\prime}, \infty}(\Omega)} \leq c,
\end{aligned}
$$

that is, (4.4).

\footnotetext{
${ }^{1}$ From now on $c$ will denote a constant which depends only on the data of the problem, but which does not depend on $\varepsilon$ and which can vary from line to line.
} 
We now turn to inequality (4.5). We observe that (4.20) holds true also with $m=m_{1}$. Therefore by Lemma 4.1, and the estimates (4.56) and (4.57), we get

$$
\begin{aligned}
\| \mid & \left.S_{m_{1}}\left(u_{\varepsilon}\right)\right|^{p-1} \|_{L^{\frac{N}{N-p}}, \infty}(\Omega) \\
\leq & C(N, p)\left[M+|\Omega|^{\frac{1}{p^{*}}} L^{\frac{1}{p^{\prime}}}\right] \\
= & C(N, p)\left[\frac{1}{C_{1}}\left\|b_{0}\right\|_{L^{N, 1}\left(Z_{\varepsilon} \cap\left\{\left|u_{\varepsilon}\right|>m\right\}\right)}\left\|\left|\nabla S_{m_{1}}\left(u_{\varepsilon}\right)\right|^{p-1}\right\|_{L^{N^{\prime}}, \infty}(\Omega)\right. \\
& \left.+\frac{1}{C_{1}} M_{0}+|\Omega|^{\frac{1}{p^{*}}} L_{1}^{\frac{1}{p^{\prime}}} m_{1}^{p-1}+|\Omega|^{\frac{1}{N^{\prime}}}-\frac{1}{p^{\prime}} L_{0}^{\frac{1}{p^{\prime}}}\right] \leq c,
\end{aligned}
$$

where $M, L, M_{0}, L_{0}$ and $L_{1}$ are defined by (4.21) and (4.22) respectively.

Moreover, we have

$$
\begin{aligned}
\left|u_{\varepsilon}\right|^{p-1} & =\left|u_{\varepsilon}\right|^{p-1} \chi_{\left\{\left|u_{\varepsilon}\right| \leq m_{1}\right\}}+\left|u_{\varepsilon}\right|^{p-1} \chi_{\left\{\left|u_{\varepsilon}\right|>m_{1}\right\}} \\
& =\left|T_{m_{1}}\left(u_{\varepsilon}\right)\right|^{p-1}+\left|S_{m_{1}}\left(u_{\varepsilon}\right)\right|^{p-1}
\end{aligned}
$$

and therefore, by the generalized Sobolev inequality (2.6), (4.58) and (4.59),

$$
\begin{aligned}
& \left\|\left|u_{\varepsilon}\right|^{p-1}\right\|_{L^{\frac{N}{N-p}, \infty}(\Omega)}=\left\|\left.\left|T_{m_{1}}\left(u_{\varepsilon}\right)\right|^{p-1}\left|\left\|_{L^{\frac{N}{N-p}, \infty}(\Omega)}+\right\|\right| S_{m_{1}}\left(u_{\varepsilon}\right)\right|^{p-1} \mid\right\|_{L^{\frac{N}{N-p}}, \infty}(\Omega) \\
& \leq c\left\|\nabla T_{m_{1}}\left(u_{\varepsilon}\right)\right\|_{\left(L^{p}(\Omega)\right)^{N}}+c \leq c,
\end{aligned}
$$

that is, 4.5.

Now we prove Theorem 4.2 when assumption 2) in Theorem 3.1 is satisfied, i.e. $\gamma<\lambda=p-1$ and $c_{0}$ belongs to $L^{\frac{N}{p-1}, \infty}(\Omega)$. We just observe that, under such assumptions, the proof made in the first case works exactly in the same way without any restriction on $\left\|c_{0}\right\|_{L^{\frac{N}{p-1}, \infty}(\Omega)}$ because $\gamma$ is less than $p-1$.

Remark 4.3. In the proof of Theorem 4.2, when $\gamma=p-1$, we use a more general assumption on the summability of $c_{0}$ (4.6), that is, $c_{0} \in L^{\frac{N}{p-1}, \infty}(\Omega)$ with $\left\|c_{0}\right\|_{L^{\frac{N}{p-1}, \infty}(\Omega)}$ small enough, and not that $c_{0} \in L^{\frac{N}{p-1}, r}(\Omega), r<\infty$, with $\left\|c_{0}\right\|_{L^{\frac{N}{p-1}, r}(\Omega)}$ small enough, as in the statement of Theorem 3.1 (see assumption 1)). This more restrictive assumption in Theorem 3.1 (which is an existence result) is due to our method which uses the stability result of Theorem 5.1 in [GM which needs $c_{0} \in L^{\frac{N}{p-1}, r}(\Omega), r<\infty$, when $\gamma=p-1$.

4.2. Passing to the limit in the approximated problem. To conclude the proof of Theorem 3.1 we have to pass to the limit in the approximated problem (3.14). This is done exactly as in Section 5 of [GM] (cf. [BMMP3]). We repeat the same arguments here for the sake of completeness.

The solution $u_{\varepsilon}$ of (3.14) satisfies

$$
\left\{\begin{array}{l}
-\operatorname{div}\left(a\left(x, u_{\varepsilon}, \nabla u_{\varepsilon}\right)+K_{\varepsilon}\left(x, u_{\varepsilon}\right)\right)=\Phi_{\varepsilon}-\operatorname{div}(g)+\operatorname{div}(F) \quad \text { in } \mathcal{D}^{\prime}(\Omega), \\
u_{\varepsilon} \in W_{0}^{1, p}(\Omega)
\end{array}\right.
$$

where

$$
\left\{\begin{array}{l}
\Phi_{\varepsilon}=f_{\varepsilon}-H_{\varepsilon}\left(x, u_{\varepsilon}, \nabla u_{\varepsilon}\right)-G_{\varepsilon}\left(x, u_{\varepsilon}\right)+\lambda_{\varepsilon}^{\oplus}-\lambda_{\varepsilon}^{\ominus} \\
\text { is bounded in } L^{1}(\Omega) .
\end{array}\right.
$$

On the one hand, using the growth condition (3.9) on $H_{\varepsilon}$ and $G_{\varepsilon}$, Theorem 4.2 and the generalized Hölder inequality (2.4), we get

$$
\left\|H_{\varepsilon}\left(x, u_{\varepsilon}, \nabla u_{\varepsilon}\right)\right\|_{L^{1}(\Omega)} \leq c
$$


and

$$
\left\|G_{\varepsilon}\left(x, u_{\varepsilon}\right)\right\|_{L^{1}(\Omega)} \leq c .
$$

On the other hand, using $T_{k}\left(u_{\varepsilon}\right)$ as a test function in (4.60), since the norm of $\left\|c_{0}\right\|_{L^{\frac{N}{p-1}, \infty}(\Omega)}$ is small enough, we easily obtain that for some $M$ and $L$, we have

$$
\int_{\Omega}\left|\nabla T_{k}\left(u_{\varepsilon}\right)\right|^{p} \leq M k+L
$$

for every $k>0$ and every $\varepsilon>0$.

Such an estimate and the growth condition (3.7) on $K_{\varepsilon}$ allow us to use standard techniques (cf. [BMu, BG2, DMOP]) to extract a subsequence of $u_{\varepsilon}$ still indexed by $\varepsilon$, such that

$$
\begin{cases}u_{\varepsilon} \rightarrow u & \text { almost everywhere in } \Omega, \\ \nabla u_{\varepsilon} \rightarrow \nabla u & \text { almost everywhere in } \Omega, \\ \nabla T_{k}\left(u_{\varepsilon}\right) \rightarrow \nabla T_{k}(u) & \text { in }\left(L^{p}(\Omega)\right)^{N} \text { weakly, }\end{cases}
$$

for every fixed $k \in \mathbb{N}$, where $u$ is a function which is measurable on $\Omega$, almost everywhere finite and such that $T_{k}(u) \in W_{0}^{1, p}(\Omega)$ for every $k \in \mathbb{N}$, with a gradient $\nabla u$ as introduced in (2.18).

By (4.63) and by the Fatou lemma, we deduce that

$$
\int_{\Omega}\left|\nabla T_{k}(u)\right|^{p} \leq M k+L
$$

and Lemma 4.1 gives

$$
|u|^{p-1} \in L^{\frac{N}{N-p}, \infty}(\Omega) \text { and }|\nabla u|^{p-1} \in L^{\frac{N}{N-1}, \infty}(\Omega) .
$$

From (4.64) and the definition (3.5) of $H_{\varepsilon}$, we deduce that

$$
H_{\varepsilon}\left(x, u_{\varepsilon}, \nabla u_{\varepsilon}\right) \rightarrow H(x, u, \nabla u) \text { almost everywhere in } \Omega .
$$

Moreover, using the growth condition (3.9) on $H_{\varepsilon}$, Theorem 4.2 and the generalized Hölder inequality (2.4), we can prove that

$$
H_{\varepsilon}\left(x, u_{\varepsilon}, \nabla u_{\varepsilon}\right) \text { is equi-integrable. }
$$

Therefore the Vitali Theorem implies that

$$
H_{\varepsilon}\left(x, u_{\varepsilon}, \nabla u_{\varepsilon}\right) \rightarrow H(x, u, \nabla u) \text { in } L^{1}(\Omega) \text { strongly. }
$$

In a similar way we prove that

$$
G_{\varepsilon}\left(x, u_{\varepsilon}\right) \rightarrow G(x, u) \text { in } L^{1}(\Omega) \text { strongly. }
$$

Therefore the solution $u_{\varepsilon}$ of (3.14) satisfies (4.66) $\left\{\begin{array}{l}-\operatorname{div}\left(a\left(x, u_{\varepsilon}, \nabla u_{\varepsilon}\right)+K_{\varepsilon}\left(x, u_{\varepsilon}\right)\right)=f_{\varepsilon}-\Psi_{\varepsilon}-\operatorname{div}(g)+\operatorname{div}(F)+\lambda_{\varepsilon}^{\oplus}-\lambda_{\varepsilon}^{\ominus} \quad \text { in } \mathcal{D}^{\prime}(\Omega), \\ u_{\varepsilon} \in W_{0}^{1, p}(\Omega),\end{array}\right.$ where $u_{\varepsilon}$ satisfies (4.64) and

$$
\begin{array}{r}
\Psi_{\varepsilon}=H_{\varepsilon}\left(x, u_{\varepsilon}, \nabla u_{\varepsilon}\right)+G_{\varepsilon}\left(x, u_{\varepsilon}\right) \rightarrow H(x, u, \nabla u)+G(x, u) \\
\text { in } L^{1}(\Omega) \text { strongly },
\end{array}
$$

where $g \in\left(L^{p^{\prime}}(\Omega)\right)^{N}$ and where $f_{\varepsilon}, \lambda_{\varepsilon}^{\oplus}$ and $\lambda_{\varepsilon}^{\ominus}$ satisfy (3.1), (3.2) and (3.3). 
Since $u_{\varepsilon}$ is a weak solution of (4.66), it is also a renormalized solution of (4.66). Therefore we can apply the stability result in [GM], which is an extension of Theorem 3.2 proved in $\mathrm{DMOP}$ when $K(x, s)=0$ (see also $\mathrm{MP}$ ). It follows that $u$ is a renormalized solution of

$\left\{\begin{array}{lr}-\operatorname{div}(a(x, u, \nabla u)+K(x, u))+H(x, u, \nabla u)+G(x, u)=f-\operatorname{div}(g)+\mu_{s}^{+}-\mu_{s}^{-} & \text {in } \Omega, \\ u=0 & \text { on } \partial \Omega .\end{array}\right.$

The proof of Theorem 3.1 is completed.

Remark 4.4. Observe that we could prove an existence result in the case where $\gamma=p-1,\left\|c_{0}\right\|_{L^{\frac{N}{p-1}, \infty}(\Omega)}$ is small enough and $\mu=f-\operatorname{div}(g)$ is a measure in $M_{0}(\Omega)$ (and not a more general measure). Indeed, under such assumptions, the a priori estimates given by Theorem 4.2 and the stability result used in Section 5 of GM still hold true (see Remark 4.3 and also Remarks 4.2 and 4.7 in [GM])

\section{ACKNOWLEDGEMENT}

This work was partially supported by GNAMPA-INdAM, Progetto "Proprietá analitico-geometriche di soluzioni di equazioni ellittiche e paraboliche" (2003). It was done during the visits made by the first author to Dipartimento di Matematica e Applicazioni "R. Caccioppoli" dell' Università degli Studi di Napoli "Federico II" and by the second author to Laboratoire de Mathématiques "Raphaël Salem" de l' Université de Rouen. Hospitality and support of all these institutions are gratefully acknowledged.

\section{REFERENCES}

[ALT] A. Alvino, P.-L. Lions, G. Trombetti, On optimization problems with prescribed rearrangements, Nonlinear Anal. 13 (1989), 185-220. MR0979040 (90c:90236)

[BGu1] M. Ben Cheikh Ali, O. Guibé, Résultats d'existence et d'unicité pour une classe de problèmes non linéaires et non coercifs, C. R. Acad. Sci. Paris 329 (1999), 967-972. MR.1733903 (2001i:35080)

[BGu2] M. Ben Cheikh Ali, O. Guibé, Nonlinear and non-coercive elliptic problems with integrable data, Adv. Math. Sci. Appl. 16 (2006), no. 1, 275-297. MR2253236 (2007d:35113)

[BBGGPV] P. Bénilan, L. Boccardo, T. Gallouët, R. Gariepy, M. Pierre, J.L. Vazquez, An L $L^{1}$ theory of existence and uniqueness of solutions of nonlinear elliptic equations, Ann. Scuola Norm. Sup. Pisa Cl. Sci. 22 (1995), 241-273. MR.1354907(96k:35052)

[BMMP1] M.F. Betta, A. Mercaldo, F. Murat, M.M. Porzio, Existence and uniqueness results for nonlinear elliptic problems with a lower order term and measure datum, C. R. Math. Acad. Sci. Paris 334 (2002), 757-762. MR1905035(2003d:35079)

[BMMP2] M.F. Betta, A. Mercaldo, F. Murat, M.M. Porzio, Uniqueness of renormalized solutions of nonlinear elliptic equations with a lower order term and right-hand side in $L^{1}(\Omega)$, ESAIM Control Optim. Calc. Var. 8 (2002), 239-272. Special issue dedicated to the memory of Jacques-Louis Lions. MR 1932952 (2003j:35082)

[BMMP3] M.F. Betta, A. Mercaldo, F. Murat, M.M. Porzio, Existence of renormalized solutions to nonlinear elliptic equations with a lower-order term and right-hand side a measure, J. Math. Pures Appl. 82 (2003), 90-124. MR.1967494

[BMMP4] M.F. Betta, A. Mercaldo, F. Murat, M.M. Porzio, Uniqueness results for nonlinear elliptic equations with a lower order term, Nonlinear Anal. 63 (2005), 153-170. MR2165494

[B] L. Boccardo, Some Dirichlet problems with lower order terms in divergence form, Preprint.

[BG1] L. Boccardo, T. Gallouët, Nonlinear elliptic and parabolic equations involving measure data, J. Funct. Anal. 87 (1989), 149-169. MR1025884 (92d:35286) 
[BG2] L. Boccardo, T. Gallouët, Nonlinear elliptic equations with right-hand side measure, Comm. Partial Differential Equations 17 (1992), 641-655. MR1163440 (94c:35083)

[BGO] L. Boccardo, T. Gallouët, L. Orsina, Existence and uniqueness of entropy solutions for nonlinear elliptic equations with measure data, Ann. Inst. H. Poincaré Anal. non linéaire 13 (1996), 539-551. MR1409661 (97f:35063)

[BMu] L. Boccardo, F. Murat, Almost everywhere convergence of the gradients of solutions to elliptic and parabolic equations, Nonlinear Anal. 19 (1992), 581-597. MR1183665 (93h:35061)

[CR] K.L. Chong, N.M. Rice, "Equimeasurable rearrangements of functions", Queen's University, 1971 (Queen's papers in pure and applied mathematics, 28). MR0372140 $(51: 8357)$

[DMOP] G. Dal Maso, F. Murat, L. Orsina, A. Prignet, Renormalized solutions for elliptic equations with general measure data, Ann. Scuola Norm. Sup. Pisa Cl. Sci. 28 (1999), 741-808. MR 1760541 (2001d:35190)

[D] T. Del Vecchio, Nonlinear elliptic equations with measure data, Potential Anal. 4 (1995), 185-203. MR1323826 (96a:35047)

[DP] T. Del Vecchio, M.M. Porzio, Existence results for a class of noncoercive Dirichlet problems, Ricerche Mat. 44 (1995), 421-438. MR.1469712 (98e:35067)

[DPo1] T. Del Vecchio, M.R. Posteraro, Existence and regularity results for nonlinear elliptic equations with measure data, Adv. Differential Equations 1 (1996), 899-917. MR1392010 (97b:35065)

[DPo2] T. Del Vecchio, M.R. Posteraro, An existence result for nonlinear and noncoercive problems, Nonlinear Anal. 31 (1998), 191-206. MR.1487540 (98k:35067)

[Dr] J. Droniou, Non-coercive linear elliptic problems, Potential Anal. 17 (2002), 181-203. MR.1908676 (2003e:35060)

[FST] M. Fukushima, K. Sato, S. Taniguchi, On the closable part of pre-Dirichlet forms and finite support of the underlying measures, Osaka J. Math. 28 (1991), 517-535. MR1144471 (93e:31012)

[G1] O. Guibé, Remarks on the uniqueness of comparable renormalized solutions of elliptic equations with measure data, Ann. Mat. Pura Appl. 180 (2002), 441-449. MR:1877627 (2003g:35067)

[G2] O. Guibé, Sur une classe de problèmes elliptiques non coercifs, to appear.

[GM] O. Guibé, A. Mercaldo, Existence and stability results for renormalized solutions to noncoercive nonlinear elliptic equations with measure data, Potential Anal. 25 (2006), no. 3, 223-259. MR2255346

[H] R. Hunt, On $L(p, q)$ spaces , Enseignement Math. 12 (1966), 249-276. MR0223874 (36:6921)

[K] B. Kawohl, "Rearrangements and convexity of level sets in P.D.E.", Springer, Berlin, New York, 1985 (Lectures Notes in Math., 1150). MR0810619(87a:35001)

[L] J.-L. Lions, "Quelques méthodes de résolution des problèmes aux limites non linéaires", Dunod et Gauthier-Villars, Paris, 1969. MR0259693 (41:4326)

[LM] P.-L. Lions, F. Murat, Solutions renormalisées d'équations elliptiques non linéaires, to appear.

[Lo] G. Lorentz, Some new functional spaces, Ann. of Math. 51 (1950), 37-55. MR0033449 $(11: 442 \mathrm{~d})$

[MP] A. Malusa, A. Prignet, Stability of renormalized solutions of elliptic equations with measure data, Atti Semin. Mat. Fis. Univ. Modena Reggio Emilia 52 (2004), no. 1, 151-168 (2005). MR2151089 (2006a:35092)

[M1] F. Murat, Soluciones renormalizadas de EDP elipticas no lineales, Preprint 93023, Laboratoire d'Analyse Numérique de l'Université Paris VI (1993).

[M2] F. Murat, Équations elliptiques non linéaires avec second membre $L^{1}$ ou mesure, Actes du 26ème Congrès National d'Analyse Numérique, Les Karellis, France, (1994), A12-A24.

[O] R. O'Neil, Integral transform and tensor products on Orlicz spaces and $L(p, q)$ spaces, J. Analyse Math. 21 (1968), 1-276. MR0626853 (58:30125)

[P] A. Prignet, Remarks on existence and uniqueness of solutions of elliptic problems with right-hand side measures, Rend. Mat. Appl.15 (1995), 321-337. MR1362776 (96j:35044) 
[S] J. Serrin, Pathological solutions of elliptic differential equations, Ann. Scuola Norm. Sup. Pisa Cl. Sci. 18 (1964), 385-387. MR0170094 (30:335)

[St] G. Stampacchia, Le problème de Dirichlet pour les équations elliptiques du second ordre à coefficients discontinus, Ann. Inst. Fourier (Grenoble) 15 (1965), 189-258. MR0192177 (33:404)

Laboratoire de Mathématiques Raphä̈l Salem, UMR 6085 CNRS, Université de Rouen, Avenue de l'Université BP.12, 76801 Saint Etienne du Rouvray, France

E-mail address: Olivier.Guibe@univ-rouen.fr

Dipartimento di Matematica e Applicazioni "R. Caccioppoli", Università degli Studi di Napoli "Federico II", Complesso Monte S. Angelo, via Cintia, 80126 Napoli, Italy

E-mail address: mercaldo@unina.it 